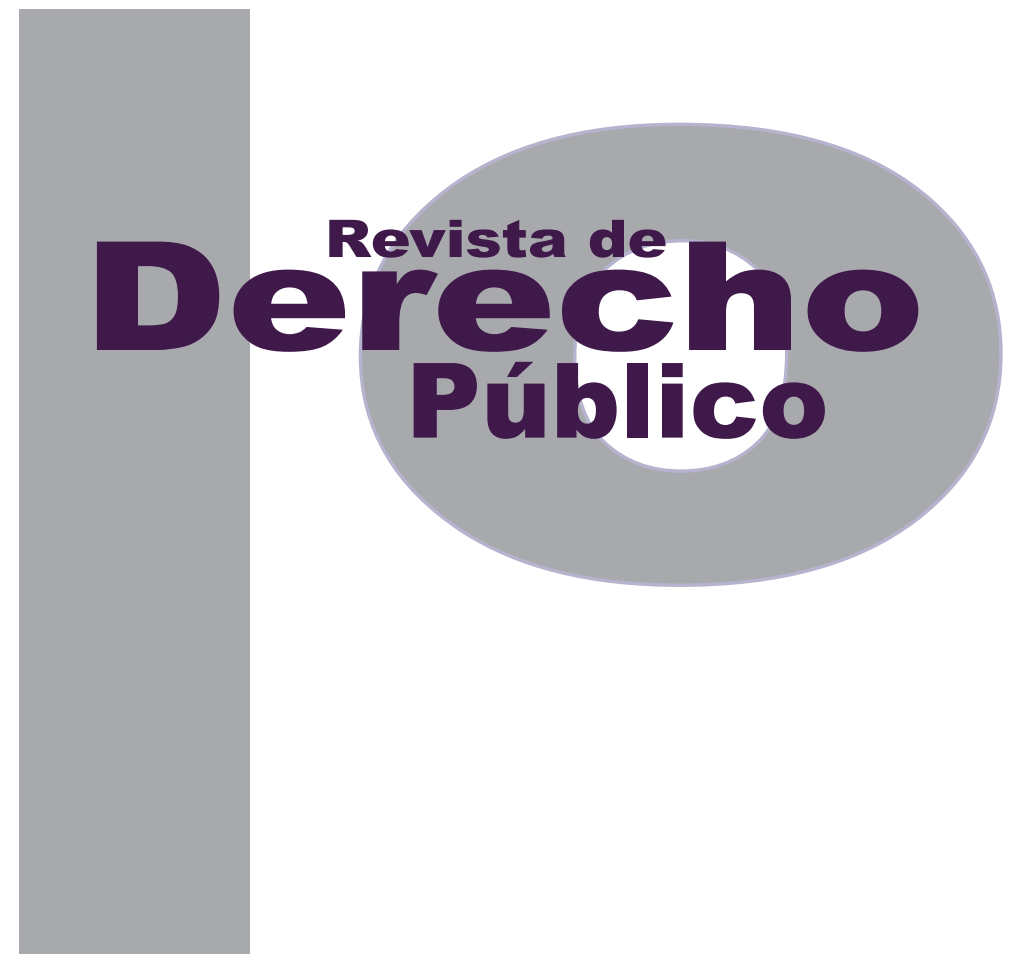

\title{
LOS DESECHOS ESPACIALES Y SU TRATAMIENTO EN EL DERECHO DEL ESPACIO ULTRATERRESTRE
}

\author{
MARÍA CAMILA IANNINI MARTíneZ
}

Universidad de los Andes

Facultad de Derecho

Revista de Derecho Público N. ${ }^{\circ} 29$

Julio - Diciembre de 2012. ISSN 1909-7778 


\title{
Los desechos espaciales y su tratamiento en el derecho del espacio ultraterrestre
}

\author{
María Camila lannini Martínez
}

\begin{abstract}
In more than 50 years of space activity, space debris has been generated around earth. Proliferation of this debris has been increasing exponentially, increasing the risk of harming earth and outer space. Although the United Nations has established, through Space Treaty, the Liability Convention and the Registration Convention, some parameters in order for the victims of the damaged caused by space debris to recognize the liability and to receive a full compensation; due to the time in which these agreements were drafted, and the amplitude in the definitions within them, it was never stipulated if the damaged caused by space debris could be regulated under these international instruments; which is why it is necessary to regulate the mechanisms used to mitigate the debris and to dissolve any type of disputes generated by the liability due to the damaged caused by space debris as in earth or in outer space.
\end{abstract}

RESUMEN
Los más de cincuenta años de actividad espacial han generado gran cantidad desechos espaciales que orbitan alrededor de la Tierra. La proliferación de estos desechos ha aumentado de manera exponencial incrementando el riesgo de causar daños en la Tierra y en el espacio ultraterrestre. Las Naciones Unidas, por medio del Tratado del Espacio, el Convenio sobre Responsabilidad y el Convenio de Registro, estableció parámetros para que las víctimas de daños causados por objetos espaciales puedan reconocer el responsable y recibir de este una indemnización pronta e integral, sin embargo, debido al momento en que estos fueron redactados y a la amplitud en sus definiciones, no se estipuló si los daños causados por desechos espaciales podrían ser regulados bajo estos instrumentos internacionales. Por esta razón es necesario reglamentar los mecanismos de mitigación de desechos y solución de disputas que se generen

Candidata a grado de Derecho en el mes de agosto de 2012, Universidad de los Andes. Alumna del curso de Derecho del Espacio Ultraterrestre en la misma universidad. Correo: mc.iannini125@uniandes.edu.co 
KEYWORDS: space object, space debris, liability, por la responsabilidad frente a los daños ocasiodamage, launching State, State of registry, miti- nados por desechos, tanto en la Tierra como en gation, nuclear energy, UnCOPOUS, NASA, ESA, orbit, el espacio ultraterrestre.

International Law, International Space Law.

Palabras clave: objeto espacial, desecho espacial, responsabilidad, daño, Estado de lanzamiento, Estado de registro, mitigación, energía nuclear, UNCOPOUS, NASA, ESA, órbita, derecho internacional, derecho espacial. 


\section{SUMARIO}

Introducción - I. DESECHOS ESPACIALES: ASPECTOS TÉCNICOS - A. Definición de desecho espacial - 1. Clases de órbitas - B. Clasificación de los desechos espaciales - C. Medición de desechos espaciales - D. Gravedad del problema - E. Desechos espaciales con carga nuclear - II. REGULACIONES Y POSIBLES SOLUCIONES - A. Diferentes regulaciones sobre desechos espaciales - $B$. Posibles soluciones técnicas - III. EL DERECHO FRENTE AL PROBLEMA DE LOS DESECHOS ESPACIALES - A. Desechos espaciales y Tratado sobre los principios que deben regir las actividades de los Estados en la exploración y utilización del espacio ultraterrestre, incluso la Luna y otros cuerpos celestes - B. Convenio sobre el Registro de Objetos lanzados al espacio ultraterrestre - C. Desechos espaciales y el Convenio sobre la responsabilidad internacional por daños causados por objetos espaciales - 1. Responsabilidad civil - 2. Responsabilidad internacional de los Estados 3. Responsabilidad espacial - 4. Responsabilidad espacial objetiva - 5. Responsabilidad espacial subjetiva - 6. Análisis de la responsabilidad espacial y los desechos espaciales - IV. CONCLUSIONES - Bibliografía. 


\section{Introducción}

A lo largo de la historia, diferentes civilizaciones se han preguntando sobre el funcionamiento de la Tierra y el espacio ultraterrestre. Dicho interés hizo que los griegos observaran el océano cósmico y bautizaran los objetos estáticos que

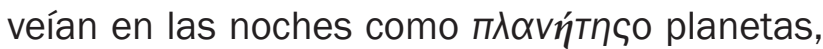
palabra que en griego significa errante ${ }^{1}$, influenciando igualmente a la filosofía puesto que al observar el espacio encontraban en él un elemento primero de todas las cosas, el origen, esencia o causa de la realidad o cosmos. ${ }^{2}$

Al transcurrir los siglos el hombre desarrolló la ciencia y con ella los principios de la física, materia que facilitaría la comprensión y estudio de los fenómenos ocurridos en la Tierra y asimismo de aquellos sucedidos en el espacio ultraterrestre. Grandes personajes como lo fueron Galileo Galilei, Johannes Kepler, Isaac Newton y Albert Einstein desarrollaron los principios científicos que en el futuro ayudaron en la comprensión de la naturaleza del espacio.

El paso del tiempo y los avances tecnológicos hicieron que la ciencia espacial se fuera perfeccionando y que aquello que parecía provenir de un libro de ciencia ficción se convirtiera en una realidad. En este orden de ideas, fue el desarrollo de la cohetería, aplicado a los misiles como armas de guerra durante las primeras décadas

1 Real Academia de la Lengua española. Recuperado el 14 de febrero de 2012 http://buscon.rae.es/drael/SrvltConsulta?TIPO_ BUS=3\&LEMA=planeta

2 Ferguson, K. 2000. La medida del universo: la histórica búsqueda de maneras para cuantificar el espacio. Ed. Robinbook. Barcelona, España. del siglo XX, liderado por los científicos Konstantin Tsiolkovsky de Rusia, Robert H. Goddard de EE.UU y Hermann Oberthy Wernher von Braun de Alemania, lo que permitió que el hombre pudiera viajar al espacio con el antecedente de la creación del misil V-2 y el cohete Saturno V que llevó al hombre a la Luna. ${ }^{3}$ Fue entonces hasta la Segunda Guerra Mundial que las potencias se encargarían de perfeccionar la cohetería, la cual llegó a su cúspide en el periodo de la guerra fría liderado por EE.UU. y la URSS.

El periodo de enfrentamiento ideológico entre el bloque occidental-capitalista (EE.UU) y oriental-comunista (URSS) contribuyó a desarrollar aún más la cohetería y la ciencia espacial. Así, la URSS lanza desde Tyuratam (Kazajistán), el primer satélite artificial, el Spútnik, el 4 de octubre de 1957, dando inicio a la era de la exploración espacial; posteriormente, el 12 de abril de 1961, lanza al espacio la primera nave espacial tripulada -Vostok 1-que puso en órbita el primer astronauta: Yuri Gagarin. Tal y como era previsto, dichos lanzamientos generaron aún más rivalidad y competencia entre las potencias por conquistar el espacio. ${ }^{4}$ Lo que siguió fue una

3 La cohetería durante la Segunda Guerra Mundial fue implementada con fines netamente bélicos y debido a las catastróficas condiciones en las cuales quedaron Europa y Japón, los miembros de la comunidad internacional reflexionaron sobre lo ocurrido y establecieron en los artículos III y IV del Tratado sobre los principios que deben regir las actividades de los Estados en la exploración y utilización del espacio ultraterrestre, incluso la Luna y otros cuerpos celestes (1967), la relevancia del mantenimiento de la paz y la utilización del espacio ultraterrestre con fines pacíficos.

4 Es pertinente aclarar que con anterioridad a la ratificación, en 1967, del Tratado sobre los principios que deben regir las actividades de los Estados en la exploración y utilización del espacio ultraterrestre, incluso la Luna y otros cuerpos celestes, en el cual se establece que el espacio ultraterrestre es inapropiable y por tal pertenece a toda la humanidad (entre otros principios), las potencias creian dar hegemonía a sus naciones apropiándose de este. 
gran serie de lanzamientos por parte de las dos potencias, en la que sobresalió EE.UU. con la Apolo 11, primera misión tripulada en llegar a la superficie de la Luna, hecho que sucedió el 21 de julio de 1969.

Por la ocurrencia de estos sucesos y el gran avance tecnológico en la materia, la ciencia espacial ha creado y desarrollado diferentes tipos de satélites entre los cuales se encuentran los de telecomunicaciones, meteorológicos, de navegación, de observación de la tierra, científicos y con propósitos experimentales, entre otros. Así las cosas, a lo largo de los más de cincuenta años de actividad espacial, se han realizado más de 4.600 lanzamientos y ubicado a 12.500 satélites ${ }^{5}$ que son rastreados y orbitan alrededor de la Tierra. Los satélites tienen una vida útil en el espacio ultraterrestre y una vez esta se ha terminado pasan a ser "basura espacial". Sin embargo, dicha basura no solo se genera por el agotamiento de la vida útil de los satélites, sino también por otros factores como las partes desprendidas del vehículo lanzador una vez este ha salido de la Tierra, por colisión entre satélites o colisión entre la misma basura espacial.

De acuerdo con lo planteado anteriormente, la siguiente investigación dirigida pretende evidenciar: i) la importancia del tratamiento de cómo el problema de los desechos espaciales ha empezado a jugar un papel tan relevante en el derecho del espacio ultraterrestre, ii) la posición de la comunidad internacional frente a los desechos, iii) la carencia de normatividad jurí-

5 Inter-Agency Space Debris Coordination Committee. IADC-WD-00-03, Versión 4.0. Revisión 8, marzo 12, 2010. p. 13. dica y sus motivos, y iv) las posibles soluciones frente al problema. Por tanto, este proyecto en un primer capítulo expone la importancia actual del problema de los desechos espaciales, la definición del concepto, sus características, el sistema de medición, los estándares planteados por las agencias espaciales y el tratamiento del problema de los desechos espaciales con carga nuclear. En un segundo capítulo se expone la regulación no vinculante relativa a los desechos y las diferentes soluciones que se han venido planteando para mitigar el crecimiento exponencial de los desechos espaciales. En el tercer capítulo se plantea el tratamiento de los desechos espaciales a la luz de la responsabilidad en el derecho del espacio ultraterrestre e internacional y se sugiere una posible solución (legislación), indicando la necesidad de normas internacionales al respecto. Por último, en el cuarto capítulo se plantean las conclusiones sobre la investigación.

\section{DESECHOS ESPACIALES: ASPECTOS TÉCNICOS}

\section{A. Definición de desecho espacial}

El nombre técnico que se le ha dado a la basura dentro del espacio es el de desecho espacial ${ }^{6} \mathrm{y}$, de acuerdo con el Lineamiento de Mitigación de Desechos Espaciales adoptado por el Comité de Usos Pacíficos del Espacio Exterior, en junio 14 de 2007, que fue avalado por las Naciones Unidas en la Asamblea General de diciembre 21 de

6 También conocido en inglés como spacedebris y en francés como débrisspatiaux. Sin embargo, para el presente trabajo el término utilizado será desecho espacial. 
2007 por la Resolución $62 / 217^{7}$, son todos los objetos no funcionales hechos por el hombre, incluidos los mismos fragmentos y elementos, que orbiten alrededor de la Tierra o ingresen a la atmósfera ${ }^{8}$. A medida que la explotación del espacio ultraterrestre continúa creciendo por medio de la utilización de telecomunicaciones ${ }^{9}$, la población de desechos espaciales también va ascendiendo, incrementando la probabilidad de colisión entre ellos o entre satélites funcionales y desechos espaciales. Consecuentemente, estos desechos son una amenaza latente para los objetos espaciales colocados en órbita y para la Tierra cuando no se desintegran e ingresan a la atmósfera ocasionando daños a la superficie o al espacio aéreo. Así las cosas, resulta apropiada la implementación de medidas que atenúen los desechos espaciales y ayuden a preservar el medio ambiente del espacio ultraterrestre para futuras generaciones.

\section{Clases de órbitas}

\section{La frontera entre el espacio aéreo de la Tierra y el espacio ultraterrestre ha sido materia de dis- cordia en la comunidad internacional, puesto}

7 UNCOPuos Main Committee Report of June 15, 2007 as contained in UN Doc. A/62/20 (Annex) pp. 47-50. The Space Debris Mitigation Guidelines as adopted by the uncopuos Main Committee were endorsed by the UN General Assembly without a vote in Resolution 62/217 "International Cooperation in the Peaceful Uses of Outer Space" of December 21, 2007 (par. 26)

8 Ibid. "space debris is defined as all man-made objects, including fragments and elements thereof, in Earth orbit or re-entering the atmosphere that are nonfunctional".

9 Hoy en día, las telecomunicaciones se manejan y realizan por medio de satélites y resultan ser un punto fundamental en la sociedad actual. Estas son algunas de las cosas que requieren la utilización de un satélite: telefonía de larga distancia, telefonía celular, Internet, GPS, cambios climáticos, televisión directa, teleobservación de la Tierra, etc. que no se ha definido con exactitud. ${ }^{10} \mathrm{~A}$ pesar de ello, la teoría de $100 \mathrm{~km}$ sobre el nivel del mar es la más aceptada, especialmente porque la actividad espacial es imposible por debajo de esta altitud. ${ }^{11}$ A pesar de la discusión de la frontera entre el límite superior del espacio aéreo y el límite inferior del espacio ultraterrestre, con seguridad se sabe que los objetos y desechos espaciales orbitan ${ }^{12}$ en cuatro diferentes órbitas.

10 El Convenio para la Reglamentación de la Navegación Aérea Internacional, más conocido como Convenio de París de 1919, estableció unos parámetros básicos para la navegación de aeronaves sobre el territorio de otro Estado. No obstante, el Convenio sobre la Aviación Civil Internacional (Convenio de Chicago de 1944), tuvo la intención de actualizar la Convención de París sobre las normas de la aviación civil, reglamentando las licencias al personal, reglamento del aire, meteorología, cartas aeronáuticas, tránsito aéreo, operación de aeronaves y aeronavegabilidad, entre muchas otras cuestiones relativas a la aviación. Sin embargo, ambos convenios omitieron establecer el límite superior del espacio aéreo por lo cual, frente a la falta de reglamentación al respecto, han surgido diferentes formas de delimitación como i) delimitación basada en criterios científicos y tecnológicos, ii) delimitación arbitraria o frontera convencional y iii) delimitación basada en la perspectiva funcional, todas ellas teniendo en cuenta de una u otra manera que la actividad espacial solo puede desarrollarse aproximadamente a los $100 \mathrm{~km}$ de altitud sobre el nivel del mar (UN DOC. A/AC105/C-2/SR, párr. 2 (5 de abril de 1979) haciendo referencia al trabajo desarrollado por la Unión Soviética UN DOC.A/AC-105/C-2/L-121).

11 Williamson, M. (2006). Space: The Fragile Frontier. American Institute of Aeronautics and Astronautics, p. 32. Recuperado de la base de datos HeinOnline el 12 de abril de 2012. Por lo general, dentro de la comunidad internacional, por medio de los diferentes tratados internacionales sobre el Derecho Aéreo como Derecho del Espacio Ultraterrestre, se ha establecido que los aviones navegan puesto que el aire es un fluido y el avión debe cortar a través de dicho fluido para moverse (artículo I y II Convenio sobre la Aviación Civil Internacional) y adicionalmente, la altitud máxima alcanzada por un avión es alrededor de $80 \mathrm{~km}$ sobre el nivel del mar. Por el contrario, según las condiciones atmosféricas que se experimentan en el espacio ultraterrestre, las naves u objetos espaciales no navegan sino que se desplazan, pues a pesar del vacío que posee la materia, su poca densidad obliga a que estas tengan un medio diferente para adquirir velocidad y propulsarse. Actualmente, debido a la evolución tecnológica desarrollada hasta el momento, las naves espaciales solo pueden desplazarse a $180 \mathrm{~km}$ desde el nivel del mar.

12 Según el Diccionario de la Real Academia de la Lengua Española, una órbita es la trayectoria que, en el espacio, recorre un cuerpo sometido a la acción gravitatoria ejercida por los astros. Recuperado el 28 de abril de 2012 http://buscon.rae.es/drael/SrvltConsulta?TIPO_ BUS $=3 \&$ LEMA $=\%$ F3ribta 
La primera se denomina órbita baja, más conocida como LEO (Low Earth Orbit), la cual se encuentra entre $200 \mathrm{~km}$ y $2.000 \mathrm{~km}$ encima de la Tierra. Es catalogada como la más densa, puesto que en ella se encuentra la mayor cantidad de objetos y desechos espaciales, debido a tres ventajas que le son características: i) al ser la que más cerca se encuentra de la Tierra se necesita menos energía para colocar la carga útil en órbita ${ }^{13}$, y al necesitar menos energía requiere un vehículo de lanzamiento más pequeño y por lo tanto menos inversión de dinero, ii) debido a la cercanía del objeto espacial con la Tierra, resulta más fácil obtener imágenes con resoluciones más altas y lograr datos más precisos, y iii) el campo magnético de la Tierra ofrece a los objetos espaciales ubicados en esta órbita una protección adicional frente a las explosiones o tormentas solares. En LEO se encuentran, generalmente, los satélites de teleobservación de la Tierra.

Una segunda es la denominada órbita de los satélites geoestacionarios, conocida como GEO (Geostationary Orbit). Es la órbita más usada después de LEo y en ella los satélites deben ser situados a aproximadamente $35.786 \mathrm{~km}$ sobre la línea ecuatorial de la Tierra, en donde rotan de manera sincronizada con un punto específico de la Tierra en un periodo de 23 horas y 56 minutos. La ventaja de esta órbita es que no requiere tener múltiples antenas que rastreen el satélite mientras orbita, en razón a que su posición permite la cobertura continua de casi la mitad

13 La carga útil es la estructura que se quiere aterrizar en el espacio ultraterrestre. Así, un cohete que sale de la Tierra querrá aterrizar en el espacio ultraterrestre un satélite; dicho satélite es la carga útil del cohete. del globo terráqueo con la ayuda de otros dos satélites, siempre permanecerá visible y gira a la misma velocidad del periodo de rotación de Tierra. La mayoría de satélites de comunicación, televisión y observación climática operan en esta órbita. Sin embargo, debido a que solamente a los $35.786 \mathrm{~km}$ sobre la línea ecuatorial los satélites experimentan el fenómeno de igual velocidad de rotación que la Tierra y al ser la órbita una circunferencia de $360^{\circ}$, resulta ser un recurso natural limitado cuyo uso y manejo está en manos de la Unión Internacional de Telecomunicaciones (UIT), organismo especializado de las Naciones Unidas encargado de asignar posiciones y llevar el registro en la órbita geo y atribuir frecuencias radioeléctricas, conceptos que juntos constituyen el llamado ROE (Recurso Órbita Espectro) ${ }^{14}$. Es relevante mencionar que el registro confiere al operador del satélite un derecho de usufructo y no un derecho de propiedad, toda vez que según el artículo II del Tratado sobre los principios que deben regir las actividades de los Estados en la exploración y utilización del Espacio Ultraterrestre, incluso la Luna y otros cuerpos celestes, el espacio no es objeto de apropiación. ${ }^{15}$

14 El Estado que desee lanzar un satélite en órbita GEO deberá realizar la publicación anticipada de frecuencias radioeléctricas y el espacio que desea ocupar en la órbita. Posteriormente, la uाT analiza y distribuye la publicación anticipada a todos los miembros de la organización y en caso de que alguno tenga problemas con la frecuencia radioeléctrica o posición orbital (recurso órbita-espectro), el Estado miembro entrará en proceso de coordinación con el otro Estado para ver cómo hace compatible la comunicación. En caso que los Estados no logren ponerse de acuerdo en el proceso de coordinación, deberán acudir a la coordinación como forma alternativa de solución de controversias. Si por el contrario, los Estados logran ponerse de acuerdo en el proceso de coordinación, se efectúa el registro en la uाт y, por tanto, se le asigna una frecuencia radioeléctrica y un espacio en la órbita.

15 Artículo II. El espacio ultraterrestre, incluso la Luna y otros cuerpos celestes, no podrán ser objeto de apropiación nacional por reivindicación de soberanía, uso u ocupación, ni de ninguna otra manera. 
En tercer lugar se encuentra la órbita media más conocida por sus siglas en inglés como MEO (Medium Earth Orbit). Esta órbita se encuentra entre la órbita LEO y debajo de la GEO. En esta se encuentran los satélites de navegación satelital, tales como los sistemas de posición global (GPS, por sus siglas en inglés) o Galileo.

Por último está la órbita alta, conocida como HEO (High Earth Orbit). Esta se encuentra después de la GEO e involucra a cualquier objeto que orbite la Tierra, tal como la Luna, naves, sondas espaciales o cualquier otro satélite artificial. Esta órbita también es conocida popularmente como cementerio espacial, puesto que es a ella a donde son lanzados los satélites geoestacionarios que han comenzado a agotar su vida útil.

\section{B. Clasificación de los desechos espaciales}

Tal como se mencionó en la introducción, a lo largo de los cincuenta años de actividad espacial se han producido considerables cantidades de desechos espaciales. De acuerdo con la Oficina del Programa de Desechos Orbitales de la NASA (NASA Orbital Debris Program Office), aproximadamente orbitan alrededor de la Tierra cerca de 19.000 objetos de más de $10 \mathrm{~cm}$ de diámetro, 500.000 desechos espaciales entre 1 y $10 \mathrm{~cm}$ de diámetro y se estima que el número de partículas más pequeñas de $1 \mathrm{~cm}$ excede los 10.000.000. ${ }^{16}$ Sin embargo, hay una clasificación más acertada sobre la materia: i) los desechos espaciales relacionados con misiones

16 Johnson, N. L., Stansbery, E., Whitlock, D.O., Abercromby, K. J., SHoots, D. (2007). History of On-Orbit Satellite Fragmentations. Orbit Debris Program Office. 14th Edition. Texas, USA. p. 6. (mission-related debris) son los que se desprenden durante el funcionamiento normal de las operaciones en órbita y representan el $12 \%$ de los desechos en órbita, por lo cual constituyen una suma a tener en cuenta; ii) los desechos espaciales desprendidos de satélites (satellite break up) son aquellos destruidos por disociación, bien sea por la carga explosiva, el cuerpo del cohete, choques accidentales o destrucción intencional y actualmente constituyen el $48 \%$ de los objetos en órbita alrededor de la Tierra, sobre una población total de 12.146 objetos; iii) los desechos espaciales por eventos anormales (anomalous events) son aquellos en que se experimenta una separación anormal del vehículo lanzado y pueden ser causados por deterioro de los materiales, tales como manta térmica, escudos protectores de radiación, paneles solares o por el impacto de pequeñas partículas. La experiencia a través de los años indica que los desechos espaciales desprendidos de satélites producen considerablemente más desechos que los desechos espaciales por eventos anormales. Por último, iv) los desechos espaciales que se producen cuando los satélites dejan de funcionar porque se quedan sin combustible o energía y no es posible maniobrarlos, es decir, se agota su carga útil.

Por otro lado, el Consejo Nacional de Ciencia y Tecnología y el Comité de Investigación de Transporte y Desarrollo de los EE.UU. en el Reporte sobre desechos espaciales (1995) clasificó los desechos en tres grupos. Un primer grupo son los desechos que miden más de $10 \mathrm{~cm}$ de diámetro y son comúnmente conocidos como objetos grandes, fácilmente detectables, rastreables y catalogables. Un segundo grupo son los 
desechos que miden entre $1 \mathrm{~cm}$ y $10 \mathrm{~cm}$ de diámetro; estos representan el mayor riesgo dentro del espacio ultraterrestre porque no pueden ser rastreables ni catalogables, y dependiendo de la velocidad con que impacten otro objeto pueden llegar a generarle daños catastróficos. El tercer grupo son los desechos que miden menos de $1 \mathrm{~cm}$ de diámetro y son más comúnmente conocidos como desechos pequeños o en algunos casos microdesechos.

\section{Medición de desechos espaciales}

Todos estos desechos son monitoreados por el Sistema de Vigilancia Espacial de los EE.UU. (Space Surveillance Systems -sss), el cual se encarga de detectar, rastrear, catalogar e identificar todos los objetos (activos o inactivos) que orbitan la Tierra por medio de potentes radares, entre los que se encuentran: i) los radares de fase de selección (phased-array radars in Low Earth Orbit -LEO) que rastrean múltiples satélites simultáneamente, pueden escanear grandes áreas del espacio en fracción de segundos y detectar desechos de tamaño milimétrico en esta órbita que es en donde se encuentran más desechos espaciales actualmente ${ }^{17}$; ii) los radares convencionales (conventional radars Midium Earth Orbit - MEo) que usan antenas de detección inmóvil y de rastreo. La antena de detección funciona transmitiendo energía al radar en forma de abanico, por lo que cuando el satélite/desecho intercepta el abanico, la energía es reflejada en la antena de rastreo y provee los

17 Catálogo de objetos espaciales

\begin{tabular}{|l|l|l|l|l|l|}
\hline Tipo de órbita & LEO & MEO & GEO & Otros & Total \\
\hline Objetos catalogados & 5747 & 134 & 601 & 1447 & 7929 \\
\hline
\end{tabular}

datos orbitales; iii) los radares terrestres de profundidad electro-ópticos del Sistema de Vigilancia Espacial (Ground-Base Electro-Optical Deep Space Surveillance System -GEODss), que consisten en tres sensores de telescopios conectados a una cámara de video que irradia la señal a unos computadores, de donde las imágenes son transportadas por medio de un impulso electrónico y gravadas en una cinta magnética, de modo que pueden ser analizadas en tiempo real18; por medio de estos radares es posible detectar desechos desde $10 \mathrm{~cm}$ en adelante.

La combinación de estos tres tipos de radares permite la observación de 80.000 satélites al día, información que es procesada por la Red del Sistema de Vigilancia (Space Surveillance Network -ssN) y transmitida al Comando Espacial de los Estados Unidos (United States Space Command -usspacecom) vía satélite, fibra óptica, microondas y telefónica. Sin embargo, la NASA no es la única institución que mide, rastrea y observa los desechos espaciales. La Unión Europea (UE) por medio de la Agencia Espacial Europea (ESA, por sus siglas en inglés) también ha desarrollado otro tipo de radares que sirven para la observación y detección de desechos en el espacio. Entre ellos se encuentran el sistema alemán TIRA, por sus siglas en inglés (Tracking and Imaging Radar) el cual detecta desechos del diámetro de $2 \mathrm{~cm}$ a una distancia de 1.000 $\mathrm{km}$ y el radar EISCAT (European Incoherent Scatter Radar) ubicado en Troms $\varnothing$, Noruega, que opera con un radar de 930-мHz uHF y 225-мHz vHF y realiza mediciones ionosféricas.

18 Space Surveillance Network. United States Space Command. Recuperado el 1 de noviembre de 2011 en: http://www.au.af.mil/au/awc/ awcgate/usspc-fs/space.htm 


\section{Gravedad del problema}

Por los beneficios que aportan los satélites a la sociedad, los radares de observación y detección juegan un papel importante para continuar abriendo la frontera espacial. No obstante, debido al avanzado desarrollo en esta materia, se ha generado una gran concentración de desechos que empeora sustancialmente por choques de satélites $^{19}$, por operación de las misiones ${ }^{20}$, por eventos anormales, por desprendimiento de partes de satélites y por terminación de la vida útil de satélites. Dicha concentración de desechos termina por afectar a los objetos puestos en órbita alrededor de la Tierra por más inmensurable que sea el espacio.

Se calcula que los desechos clasificados y cuantificados por la NASA, como ya vimos, pueden viajar a una velocidad de $20 \mathrm{~km} /$ segundo y causar diferentes daños. Así, los desechos que tienen menos de $0.01 \mathrm{~cm}$ pueden ocasionar hoyos y erosión sobre otros objetos espaciales; los del tamaño de $0.01 \mathrm{~cm}$ a $1 \mathrm{~cm}$ producen impactos significativos ocasionando daños severos dependiendo de la vulnerabilidad del sistema y el diseño con el cual esté protegido el objeto

19 El primer choque de satélites ocurrió el 10 de febrero de 2009 cuando el satélite de comunicación ruso Cosmos-2251, que estaba fuera de funcionamiento desde 1995, chocó con el satélite estadounidense Iridium-33 a $776 \mathrm{~km}$ de la Tierra. El choque produjo una nube de más de 500 fragmentos de desecho espacial que se han ido dispersando. Tal como lo indicaron la nasa y esa, este fue el primer choque entre satélites que orbitaban normalmente en los más de cincuenta años de actividad espacial y que puso en alerta a la comunidad internacional por futuros acontecimientos de igual carácter. Recuperado el 30 de octubre de 2011 en: http://www.esa.int/gsp/ACT/ariadna/Ariadna\%20 Projects/ARI_study_10-Cfl.html

20 El 11 de enero de 2007 China destruyó un satélite meteorológico FENGYUN por medio de un proyectil cinético, el cual produjo gran cantidad de desechos espaciales. Recuperado el 30 octubre de 2011 en: http:// www.technologyreview.com/computing/18281/ espacial impactado, por ejemplo, el daño que puede ocasionar un desecho del tamaño de 1.3 $\mathrm{cm}$ en órbita LEo, viajando a una velocidad de $10 \mathrm{~km} / \mathrm{s}$ equivale al impacto de una bala calibre $22^{21}$ y un desecho de $1 \mathrm{~cm}$ de diámetro ubicado en esta órbita puede dañar un satélite en perfecto funcionamiento al colisionar con él ${ }^{22}$; los desechos de $10 \mathrm{~cm}$ o más al impactar otro objeto producirían efectos catastróficos puesto que existe una gran probabilidad de que dañen de manera considerable el objeto impactado o generen más desechos. Sin embargo, el número de partículas registradas de al menos $10 \mathrm{~cm}$ sigue siendo pequeña (aunque ha venido aumentando) por lo cual es improbable que una colisión ocurra. Si bien los satélites pueden ser protegidos por un escudo o blindados para salvaguardarlos de futuras colisiones, el material extra utilizado para dicho blindaje hace que el lanzamiento del vehículo, el poder maniobrarlo y su aterrizaje resulte difícil y costoso. ${ }^{23}$ Por otro lado, la gran demanda por el uso de la órbita geoestacionaria ha hecho que una vez se sepa que el satélite ha empezado a agotar su vida útil, la UIT ordene su lanzamiento a una órbita más alta como la HEO o cementerio espacial. La Tierra, observada desde afuera, se ve como se muestra en la siguiente imagen:

21 Oficina de Ciencia y Tecnología. Reporte interinstitucional sobre desechos espaciales. Noviembre de 1995. p. 8. Recuperado el 30 de octubre de 2011 en: http://orbitaldebris.jsc.nasa.gov/library/IAR_95_Document.pdf

22 Monitoring Space Debris. European Space Agency, video http://www. youtube.com/watch?v=8iRsaizBUaY. Recuperado el 20 de noviembre de 2011.

23 Pusey, N. (2010). The Case of Preserving Nothing: The Need for a Global Response to the Space Debris Problem. Colorado Journal of International Environmental Law and Policy. 425, Spring, p. 430. 


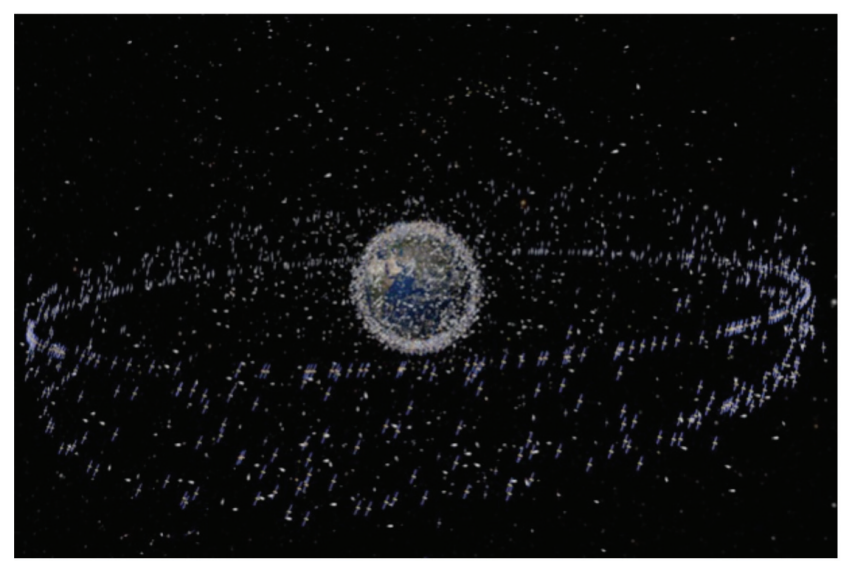

Adicionalmente, es necesario mencionar que la Tierra tiene un efecto positivo sobre muchos de los desechos espaciales. Al perder energía y dependiendo del tamaño pueden ocurrir dos cosas: que se quemen por la fricción con la parte alta de la atmósfera o que con el paso del tiempo caigan progresivamente en diferentes órbitas y finalmente entren a la atmósfera atraídos por la fuerza gravitacional de la Tierra. La cantidad de desechos que siguen orbitando es más grande que la de aquellos que ingresan a la atmósfera y posteriormente caen en la Tierra. Así se evidenció en satélites como el Cosmos 954 que cayó en Canadá en el año 1978, la estación espacial Mirque cayó en el océano Pacífico el 2001 o el satélite UARS (Upper Atmosphere Research SateIlite) que también cayó en el océano Pacífico en septiembre de 2011.

Pero los desechos no solo afectan a otros objetos espaciales, sino también la observación astronómica. En la actualidad existen "nubes de desechos" creadas por la incontrolada colisión entre los mismos desechos o por la explosión intencional de satélites, ${ }^{24}$ las cuales no permiten

24 El 11 de enero de 2007, China, realizando pruebas militares, lanzó un misil balístico de medio alcance desde el centro espacial Xichang para destruir el satélite meteorológico FENGYUN 1C, el cual se encontraba en observar con claridad el espacio ultraterrestre y, además, representan un riesgo para el lanzamiento y aterrizaje de objetos espaciales porque van creando una especie de "alambre de púas" alrededor de la Tierra.

Los desechos espaciales, como se ha evidenciado, no solo han venido aumentando sino que, como afirmara el científico Donald Kessler (1978) en su libro Frecuencia de colisión de satélites artificiales: la creación del cinturón de desechos, a medida que los satélites artificiales se incrementen en el espacio, la probabilidad de colisión entre estos también se acrecienta creando un cinturón de estos alrededor de la Tierra. Los cálculos realizados por Kessler y su teoría no resultaron del todo desacertados, puesto que si bien el autor predijo que la primera colisión sucedería entre 1986 y 1997 (ver gráfica), el primer choque de satélites se produjo el 10 de febrero del 2009 entre el Iridium 33 y el Cosmos 2251, lo que según la ssn generó grandes nubes de desechos.

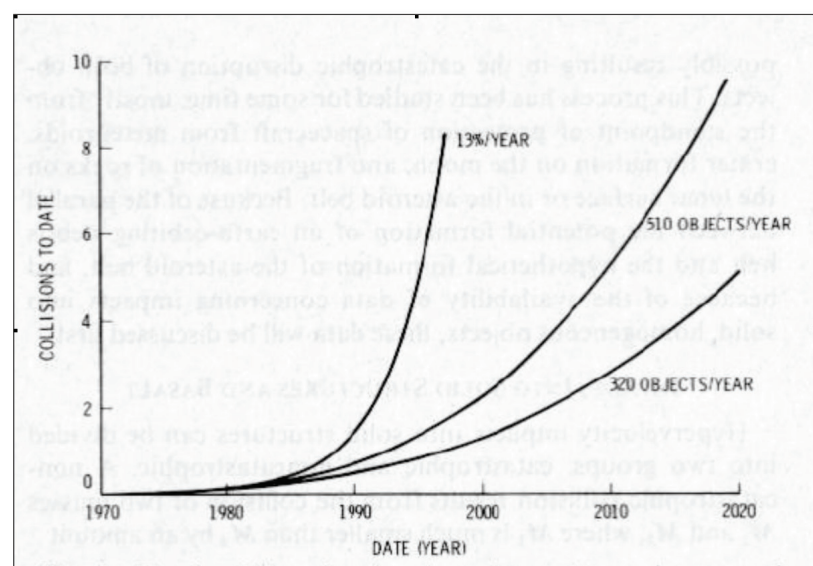

Fig. 4. Total collisions by the given date under various growth assumptions. The first collision is expected between 1989 and 1997.

órbita desde 1999 y estaba muy cerca de la Estación Espacial Internacional. La destrucción del satélite produjo tal cantidad de desechos que debieron elevar la estación 1,7 km hasta que alcanzó 391,6 km para evitar un posible desastre en caso de que los desechos chocaran con ella. 


\section{E. Desechos espaciales con carga nuclear}

Otra de las grandes preocupaciones con respecto a los desechos espaciales tiene que ver con los objetos con carga nuclear. ${ }^{25} \mathrm{Sin}$ bien para algunos científicos y expertos espaciales la energía nuclear es la clave del éxito futuro de la humanidad en el espacio, ${ }^{26}$ ambientalistas y grupos antinucleares discrepan de esta posición por las consecuencias catastróficas que la energía nuclear puede ocasionar tanto en la Tierra como en el espacio ultraterrestre.

El uso de energía nuclear en misiones espaciales se ha hecho a través de generadores radioisotópicos termoeléctricos (radioisotope thermoelectric generators - RTG), los cuales proveen energía a la nave espacial convirtiendo el calor del decaimiento natural del plutonio en electricidad, es decir, no son reactores nucleares puesto que no producen energía nuclear por fusión o fisión. ${ }^{27}$ Este tipo de energía tiene como ventaja su peso, eficiencia, longevidad, confiabilidad y durabilidad en el espacio ultraterrestre. Los RTG han sido usados desde 1960 en misiones como

25 McAvoy, J. J. (2004). Nuclear Space and the Earth Enviroment: the Benefits, Dangers and Legality of Nuclear Power and Propulsion in Outer Space. William and Mary Environmental Law and Policy Review. Vol. 29, p.193.

26 The Pro-Nuclear Space Movement. Recuperado el 1 de abril de 2012 en: http://www.nuclearspace.com/.Robert Zubrin. Power and Purpose in Space: Without Nuclear-Powered Spacecraft, We'll Never Get Anywhere, IEEESPECTRUM, en: http:/!www.spectrum.ieee.org/WEBONLY/resource/nov02/speak2.htm.

${ }^{15}$ Grossman, K. (1997). The Wrong Stuff: The Space Program's Nuclear. Common Courage Press. Atlanta Book Company, p. 45.

27 Un reactor nuclear es un dispositivo donde se produce una reacción nuclear controlada. Opera convirtiendo el calor generado por la fisión de átomos pesados en electricidad. la Apolo a la Luna, la Vikingo a Marte y las Pionero, Ulises y Galileo a otros sistemas solares. ${ }^{28}$ A pesar del éxito de las misiones antes nombradas, el fracaso de los satélites SNAP-9A RTG (1964), NimbusB-1 (1968), Cosmos 954 (1968) y la misión a la Luna, Apolo 13 (1970), han creado desconfianza en la comunidad antinuclear y protectora del medio ambiente.

De acuerdo con lo planteado con anterioridad, la creciente e importante utilización de la energía nuclear en actividades espaciales hizo que la comunidad internacional adoptara los Principios pertinentes a la utilización de las fuentes de energía nuclear en el espacio ultraterrestre, aprobados por la Asamblea General de las Naciones Unidas en la Resolución 47/68 del 14 de diciembre de 1992, en la cual se reconocen los riesgos que el material radioactivo entraña y, por tanto, dentro de sus objetivos generales establece medidas de protección contra la radiación y seguridad nuclear. Sin embargo, por el hecho de ser aprobados en una resolución y no en un tratado, estos principios no son vinculantes pero sí representan estándares internacionalmente reconocidos, pues tal como aparece en el Principio I. Aplicabilidad del derecho internacional, las actividades relativas a la utilización de fuentes de energía nuclear en el espacio ultraterrestre se efectuarán de conformidad con el derecho internacional, particularmente de conformidad con la Carta de las Naciones Unidas y el Tratado sobre los principios que deben regir las actividades de los Estados en la exploración y utilización del espacio ultraterrestre, incluso la Luna y otros cuerpos celestes.

28 Loft, K. (1999). Too Close for Comfort? The Tampa Tribune Press, p. 6 . 


\section{REGULACIONES Y POSIBLES SOLUCIONES}

\section{A. Diferentes regulaciones sobre desechos espaciales}

Debido a la gravedad del problema que representan los desechos espaciales, los países reconocen la importancia de mantener un acceso sostenible al espacio ultraterrestre para las futuras generaciones y diferentes actores (Estados, organizaciones gubernamentales y no gubernamentales) han creado ciertas recomendaciones al respecto. La NASA fue la primera agencia que, en 1995, manifestó la problemática sobre los desechos espaciales en un documento que mostraba una serie de parámetros para su mitigación; dos años después, con ayuda del Departamento de Defensa, estableció una serie de parámetros con el mismo propósito. De igual forma, agencias espaciales como la francesa, rusa, japonesa y europea han desarrollado también sus propios parámetros para la mitigación de los desechos.

Por otro lado, si bien la Oficina de Asuntos Espaciales de las Naciones Unidas (UNoosA, por sus siglas en inglés), por medio del Comité para el Uso Pacífico del Espacio Ultraterrestre (más conocido como uncopuos, por sus siglas en inglés) el cual se encuentra dividido en dos subcomités: el Comité Jurídico y el Comité Técnico y Científico, tomó nota del problema que representa la basura espacial en $1980^{29}$ y aunque desde 1994 los desechos espaciales habían sido materia

29 BAKER, H. A. (1989). Space Debris: Legal and Policy Implications. University of Colorado Law Review 55. de discusión en el Comité Técnico y Científico, es solo hasta 1999 que este publicó un reporte técnico sobre desechos espaciales reconociendo el riesgo de estos para los objetos puestos en órbita alrededor de la Tierra y directrices para aminorar tales desechos en el espacio.

Posteriormente, el 15 de octubre de 2002, se crea el Comité de Coordinación Interinstitucional de los Desechos Espaciales ${ }^{30}$ (Inter-Agency Space Debris Coordination Committee - IADC) con el objetivo de observar, investigar y mitigar los desechos en el espacio, teniendo como propósitos: i) limitar los desechos expulsados durante las operaciones normales; ii) minimización del potencial de los desprendimientos en órbita; iii) manejo de los residuos post-misiones y iv) prevención de colisiones en órbita (IADC-02-01). Adicionalmente, el IADC recomienda que la mitigación de desechos sea implementada en cualquier proyecto espacial y requiere que los Estados de manera voluntaria realicen un reporte de la misma clase con los esfuerzos adelantados para mitigar la creación de nuevos desechos.

Finalmente, en el 2007 el Comité Técnico y Científico adoptó, de manera consensual, parámetros para la mitigación de desechos espaciales. Dichos parámetros fueron subsecuentemente adoptados por el comité principal de uncopuos el 15 de junio de 2007, y apoyados por la Asamblea General de las Naciones Unidas

Este Comité está conformado por la Agencia Espacial Italiana, el Centro Británico del Espacio, el Centro Nacional de Estudios Espaciales de Francia, la Administración Nacional del Espacio Chino, el Centro Alemán del Espacio, la Agencia Espacial Europea (ESA, por sus siglas en inglés), la Organización de Investigación del Espacio India, Japón, la NASA, la Agencia Nacional del Espacio de Ucrania y la Agencia Aeronáutica y Espacial de Rusia, 
por consenso en la Resolución 61/111 "Cooperación Internacional en los Usos Pacíficos del Espacio Ultraterrestre" en febrero 15 de 2008. Este texto técnico fue redactado de manera comprensiva (sorpresivamente), explicando la filosofía de la mitigación de los desechos espaciales de manera general y estableciendo la importancia de la adopción de medidas para su mitigación. La naturaleza de estos parámetros no es vinculante para los Estados sino más bien recomendatoria, ${ }^{31}$ debido a que representan una solución al problema de la mitigación en aquellos países productores de objetos espaciales. Así, se puede evidenciar cómo EE.UU., la ESA o Japón han adoptado fuertes estándares para mitigar los desechos, los cuales son aplicados actualmente con mayor rigidez que los establecidos en uncopuos. Sin embargo, si bien el carácter de estos lineamientos resulta ser recomendatorio y no vinculante, su práctica reiterada puede convertirse en costumbre internacional.

\section{B. Posibles soluciones técnicas}

Adicional a la guía adoptada por la Asamblea General de las Naciones Unidas, las diferentes agencias espaciales han planteado posibles soluciones para mitigar los desechos espaciales. Dentro de aquellas soluciones se encuentran:

I. un láser vaporizador o redireccionador con el cual se pretende desintegrar los desechos espaciales de manera que desaparezcan del espacio ultraterrestre o redireccionarlos para que eventualmente se desintegren en la atmósfera

31 BenKö, M. and KAI-UwE, S. (2008). European Space Policy Institute. German Journal of Air and Space Law 335, p. 12. o ingresen a la Tierra. Esta solución tiene bastantes críticas porque el redireccionamiento de los desechos hacia la Tierra puede ocasionar futuros choques con objetos $u$ otros desechos espaciales produciendo mayor basura espacial;

II. crear un cinturón de desechos que orbiten alrededor de un objeto espacial, buscando así que futuros desechos no ocasionen daños a los satélites o naves espaciales, puesto que al acercarse integrarán el cinturón ya conformado por basura espacial disminuyendo el riesgo de causar daños en la carga útil del objeto sobre el cual orbitan. En cuanto a esta solución, los científicos son escépticos en cuanto a poder lograr que los desechos espaciales orbiten alrededor de un objeto creando un cinturón protector, puesto que la tecnología para generar las fuerzas gravitacionales que permitan que el objeto espacial atraiga a los desechos no se encuentra desarrollada a profundidad;

III. poner escudos protectores o blindar las naves o satélites para salvaguardarlos de futuras colisiones. Sin embargo, el material extra utilizado para dicho blindaje hace que el lanzamiento del vehículo, el poder maniobrarlo y su aterrizaje resulte difícil y costoso;

IV. un sistema de captura mediante una red. Pretende poner una nave espacial que a través de su desplazamiento vaya aprehendiendo desechos en una malla, de manera tal que estos queden atrapados en la red y no generen eventuales daños a objetos espaciales ni colisionen con otros desechos. Este sistema está siendo construido y probado por la Universidad de CoIorado en Estados Unidos y aspira capturar desechos hasta de $30 \mathrm{~cm}$ de diámetro ${ }^{32}$; 
V. un sistema de reingreso de satélites y naves espaciales a la Tierra de manera controlada, cuando estos hayan cumplido su misión en el espacio ultraterrestre. En efecto, cuando los objetos en LEo están cercanos a agotar su vida útil, estos deben ser programados para reingresar a la Tierra y no generar futura basura espacial. En los satélites que tengan poca capacidad para ser maniobrados y en vista de la incertidumbre del tiempo exacto de reingreso como también el lugar donde impactarán en la Tierra, se ha planteado la posibilidad de calcular un reingreso y caída sobre el océano, aunque la mayoría de veces su impacto será menor debido a que el satélite se quemará en gran parte al ingresar a la atmósfera ${ }^{33}$;

VI. un sistema reductor de velocidad por medio de una bola de espuma. Consiste en una plataforma capaz de lanzar una bola de espuma alrededor de un desecho específico, de tal forma que incremente su radio área-a-masa y la resistencia atmosférica ejerza una influencia significativa que lo desacelere logrando que un desecho que en principio orbitaría por cientos de años alrededor de la Tierra, logre reducir su velocidad y finalmente ingrese a la atmósfera. ${ }^{34}$ Este sistema de limpieza espacial tiene como objetivo ubicarse debajo de los $2.000 \mathrm{~km}$

Capture, and De-Orbit of Orbital Debris. Submitted to the Revolutionary Aerospace Systems Concepts Academic Linkage (RASC-AL). Conference May 27, 2011. On the theme of Orbital Debris Mitigation Approaches.

Diederiks-Verschoor, I.H. y Kopal, V. (2008). An Introduction to Space Law. Kluwer Law International. The Netherlands. Third Revised Edition, p. 130.

ANDRENUCCI, M., PERGOlA, P., RUGGIERO, A. (2011). European Space Agency. Active Removal of Space Debris, Expanding foam application for active debris removal. Final Report. February 2th "The core idea is to develop a platform able to realize a foam ball around a target debris that enlarges its area-to-mass ratio such that the atmospheric drag can exert a significant influence to decelerate the debris. In this way, debris that would have orbited for hundreds of years, will re-entry in a prescribed time", p. 10. (órbita LEO) de altura desde la Tierra puesto que allí es donde hay más densidad de desechos. De igual forma, este sistema tiene grandes ventajas: (a) al ser una esfera de espuma no necesita piezas $u$ objetos que encajen perfectamente, reduciendo los riesgos relacionados con el funcionamiento del sistema. (b) La estructura de espuma es más sólida y resistente que otras estructuras como, por ejemplo, el sistema de captura mediante red. (c) El cambio de velocidad está dado solo por la fuerza de arrastre, lo cual hace que el desecho se desacelere hasta quemarse por completo en la atmósfera. (d) La estructura de espuma está, en principio, diseñada para desacelerar desechos específicos que amenacen de forma latente a otros desechos espaciales en la órbita LEO; y por último,

VII. un sistema satélite aspiradora, el cual ha sido propuesto por el Centro Suizo del Espacio y desarrollado por la Universidad de Lausanne. Sería un satélite que a medida que recorre su órbita actúe como una "aspiradora espacial" limpiando el espacio ultraterrestre de desechos.

\section{EL DERECHO FRENTE AL PROBLEMA DE LOS DESECHOS ESPACIALES}

\section{A. Desechos espaciales y Tratado sobre los principios que deben regir las activi- dades de los Estados en la exploración y utilización del espacio ultraterrestre, incluso la Luna y otros cuerpos celestes}

Ante el papel preponderante que las actividades espaciales empezaron a jugar en la sociedad y ante los riesgos que comporta el ejercicio de estas, los Estados, por medio de las Naciones 
Unidas, decidieron consensuar y ratificar una serie de tratados (Tratado del Espacio, Convenio sobre la Responsabilidad y Convenio de Registro) a través de los cuales establecen principios fundamentales para el ejercicio de la actividad espacial y reglamentan la materia en caso de presentarse un daño. A continuación se expondrá el tratamiento de los desechos espaciales a la luz del derecho del espacio ultraterrestre e internacional y se sugerirá una posible solución (legislación) frente al tema, indicando la necesidad de normas internacionales al respecto.

Reconociendo el interés general de toda la humanidad en el proceso de exploración y utilización del espacio ultraterrestre, como también inspirados en las grandes perspectivas que se ofrecen a la humanidad como consecuencia de la entrada del hombre en el espacio ultraterrestre, los Estados, por medio de la comunidad internacional reunida a través de las Naciones Unidas, celebraron el Tratado del Espacio. En este Tratado, el artículo IX establece que “En la exploración y utilización del espacio ultraterrestre, incluso la Luna y otros cuerpos celestes, los Estados Partes en el Tratado deberán guiarse por el principio de la cooperación y la asistencia mutua, y en todas sus actividades en el espacio ultraterrestre, (...)". Asimismo, los Estados Partes del Tratado "harán los estudios e investigaciones del espacio ultraterrestre, incluso la Luna y otros cuerpos celestes, y procederán a su exploración de tal forma que no se produzca una contaminación nociva ni cambios desfavorables en el medio ambiente de la Tierra como consecuencia de la introducción en él de materias extraterrestres, y cuando sea necesario adoptarán las medidas pertinentes a tal efecto" (resaltado fuera del texto original) y si un Estado "tiene motivos para creer que una actividad o un experimento en el espacio ultraterrestre, (...) proyectado por él o por sus nacionales, crearía un obstáculo capaz de perjudicar las actividades de otros Estados Partes en el Tratado en la exploración y utilización del espacio ultraterrestre con fines pacíficos, (...) deberá celebrar las consultas internacionales oportunas antes de iniciar esa actividad o ese experimento" (resaltado fuera del texto original). Igualmente, en los artículos VI y VII se estableció que "Ios Estados Partes en el Tratado serán responsables internacionalmente de las actividades nacionales que realicen en el espacio ultraterrestre," y que "todo Estado Parte que lance o promueva el lanzamiento de un objeto al espacio ultraterrestre (...) o desde cuyo territorio o cuyas instalaciones se lance un objeto, será responsable internacionalmente de los daños causados a otro Estado Parte en el Tratado o a sus personas naturales o jurídicas por dicho objeto o sus partes componentes en la Tierra, en el espacio aéreo o en el espacio ultraterrestre, incluso la Luna y otros cuerpos celestes" (resaltado fuera del texto original).

Teniendo en cuenta lo anterior, por ser el primer tratado relacionado con actividades espaciales, estaba destinado a proporcionar parámetros generales y principios fundamentales que se esperaba fueran posteriormente desarrollados, de manera específica, por medio de otros tratados. No obstante, la vaga terminología del artículo IX demuestra la falta de avances tecnológicos y habilidades frente a la materia para ese entonces, puesto que era imposible prever la contaminación o polución del espacio ultra- 
terrestre y la posibilidad de que aquella contaminación interfiriera con la actividad espacial de los Estados. En consecuencia, el Tratado del Espacio no definió qué se debía entender por “objeto espacial” 35 como tampoco qué significaba "contaminación nociva" o el alcance de la expresión "los Estados, cuando sea necesario, adoptarán las medidas pertinentes". Tampoco estableció un parámetro para determinar cuál de perjudicar" ni los límites en las actividades o experimentos desarrollados en el espacio ultraterrestre.

A la par, en el artículo VI establece que "Ios Estados Partes en el Tratado serán responsables internacionalmente de las actividades nacionales que realicen en el espacio ultraterrestre", y en el artículo VII estatuye que cada

Estado Parte en el Tratado que lance o promueva el lanzamiento de un objeto al espacio ultraterrestre, incluso la Luna y otros cuerpos celestes, y todo Estado Parte en el Tratado, desde cuyo territorio o cuyas instalaciones se lance un objeto, será responsable internacionalmente de los daños causados a otro Estado Parte en el Tratado o a sus personas naturales o jurídicas por dicho objeto o sus partes componentes en la Tierra, en el espacio aéreo o en el espacio ultraterrestre, incluso la Luna y otros cuerpos celestes. debía ser la interpretación de "obstáculo capaz

Así, este artículo también extiende la responsabilidad a los nacionales que realicen actividades en el espacio ultraterrestre, la Luna y otros cuerpos celestes pero no establece bajo qué términos jurídicos el Estado será responsable.

Los artículos anteriores, si bien no hablan específicamente del problema de los desechos espaciales, proveen unos lineamientos para determinar de qué modo los desechos espaciales y la responsabilidad derivada de los daños pueden ser regulados. Sin embargo, no existe una definición exacta de "objeto espacial o las partes que lo componen", por lo que resulta incierto si los desechos espaciales podrían ser interpretados bajo esta legislación ${ }^{36}$. Más aún, tampoco hay certeza sobre si por el término “objeto espacial”, el daño causado por un desecho espacial puede ser atribuido a la responsabilidad de un Estado o si las actividades generadas por organizaciones espaciales no gubernamentales o agencias espaciales privadas estén de acuerdo con el concepto vago y general de lo establecido por el artículo VI. Aun más, en dicho Tratado no se determinó el tipo de responsabilidad jurídica que le sería atribuida a los Estados. Por tanto, a la luz de este Tratado la normatividad no es suficiente para abarcar los desechos espaciales y resulta necesaria una regulación específica sobre la materia.

\section{B. Convenio sobre el registro de objetos lanzados al espacio ultraterrestre}

35 No se debe confundir objetos espaciales con desechos espaciales, puesto que el primero resulta ser un bien mueble registrable, el cual tiene un uso específico en relación con la actividad espacial que desempeña, por ejemplo, un satélite o un cohete, mientras que el segundo "son todos los objetos no funcionales hechos por el hombre, incluidos los mismos fragmentos y elementos, que orbiten alrededor de la Tierra o ingresen a la atmósfera" de acuerdo con el Lineamiento para la Mitigación de Desechos Espaciales de la UNCOPOUS.
El segundo tratado que tiene estrecha relación

36 Jasentuliyana, N. (1998). Space Debris and International Law. 29 Journal of Space Law, V. 139, p. 142. 
con los desechos espaciales es el Convenio sobre el registro de objetos lanzados al espacio ultraterrestre (también conocido como Convenio de Registro), aprobado por la Asamblea General de las Naciones Unidas en su Resolución 3235 (XXIX), el 12 de noviembre de 1974, convenio en el cual se expresa el deseo de establecer un registro central de los objetos lanzados al espacio ultraterrestre con carácter obligatorio, como también el interés de suministrar a los Estados Partes medios y procedimientos adicionales para ayudar a la identificación de los objetivos espaciales, convencidos de que un sistema obligatorio de registro de los objetos lanzados al espacio ultraterrestre ayudaría a su identificación y contribuiría a la aplicación y el desarrollo del derecho internacional que rige la exploración y utilización del espacio ultraterrestre. ${ }^{37}$

Si bien es cierto que el Convenio sobre la responsabilidad internacional por daños causados por objetos espaciales (conocido como Convenio sobre la Responsabilidad) fue primero que el Convenio de Registro, es importante explicar primero este último en aras de entender más fácilmente la responsabilidad causada por objetos espaciales y las dificultades que se generan al aplicarla a los desechos espaciales. Así, el Convenio de Registro se creó en armonía con el Tratado del Espacio y el Convenio sobre Responsabilidad para resolver el problema de la identificación de los objetos en el espacio ultraterrestre, derivados de las actividades de los seres humanos.

Preámbulo del Convenio sobre el registro de objetos lanzados al espacio ultraterrestre.
En efecto, en el Convenio de Registro se adopta la definición de "Estado de lanzamiento" 38 , como también lo que ha de entenderse por "objeto espacial", el cual incluye el vehículo propulsor y sus partes, tal y como se encuentra definido en el Convenio sobre Responsabilidad. El Convenio de Registro estableció, además, que por “Estado de registro" deberá entenderse "un Estado de lanzamiento en cuyo registro se inscriba un objeto espacial de conformidad con el artículo II", es decir, que todo Estado que lance un objeto al espacio ultraterrestre debe llevar un registro nacional sobre este, y debe notificar al Secretario General de las Naciones Unidas dicho registro. Además, el mismo artículo expone que cuando haya dos o más Estados de lanzamiento, entre ellos deben decidir cuál de los dos llevará el registro del objeto espacial. El registro debe contener: i) nombre del Estado o de los Estados de lanzamiento; ii) una designación apropiada del objeto espacial o su número de registro; iii) fecha y territorio o lugar de lanzamiento; iv) Parámetros orbitales básicos; y, v) función general del objeto espacial. ${ }^{39}$ Lo anterior con el fin de que la información sea pública para todos los Estados Partes del tratado y que, en caso de generarse un daño por un objeto espacial, el Estado responsable sea identificable para el Estado al que se le causó el perjuicio y pueda iniciar tan pronto como le sea posible una reclamación. ${ }^{40}$

38 Artículo I a). Se entenderá por "Estado de lanzamiento": i) Un Estado que lance o promueva el lanzamiento de un objeto espacial; ii) Un Estado desde cuyo territorio o desde cuyas instalaciones se lance un objeto espacial.

39 Artículo IV del Convenio sobre Registro.

40 Manrique, Ana María y Morales, Valentina. (2012). Responsabilidad internacional por daños causados por objetos espaciales. Tesis no publicada. Universidad de los Andes, Bogotá, Colombia, p. 38. 
De igual forma, el Convenio de Registro obliga al Estado de lanzamiento a que registre dichos objetos ante la Secretaría General de la Naciones Unidas, en cuanto sea factible la información sobre cada objeto espacial que haya sido lanzado (artículo IV) ${ }^{41}$. Desafortunadamente, la forma en que fue redactado el artículo IV hace que la información que tiene que proveer el Estado de registro sea limitada (no suficiente) y que se presente ambigüedad interpretativa cuando se afirma que "todo Estado de registro proporcionará al Secretario General de las Naciones Unidas, en cuanto sea factible, [...] información sobre cada objeto espacial"42 (resaltado fuera del texto original). Esta cuestión ha hecho que el Convenio de Registro no haya sido seriamente usado como una herramienta para mantener el registro de objetos espaciales, pues muchos Estados omiten información al considerar que no es "factible" suministrarla ${ }^{43}$. Adicionalmente, la forma en la que el Convenio fue redactado no permite tener una clara interpretación sobre si solo los satélites activos son los que deben

41 Artículo IV 1. Todo Estado de registro proporcionará al Secretario General de las Naciones Unidas, en cuanto sea factible, la siguiente información sobre cada objeto espacial inscrito en su registro: a) Nombre del Estado o de los Estados de lanzamiento; b) Una designación apropiada del objeto espacial o su número de registro; c) Fecha y territorio o lugar del lanzamiento; d) Parámetros orbitales básicos, incluso: i) Período nodal; ii) Inclinación; iii) Apogeo; iv) Perigeo; e) Función general del objeto espacial. 2. Todo Estado de registro podrá proporcionar de tiempo en tiempo al Secretario General de las Naciones Unidas información adicional relativa a un objeto espacial inscrito en su registro. 3. Todo Estado de registro notificará al Secretario General de las Naciones Unidas, en la mayor medida posible y en cuanto sea factible, acerca de los objetos espaciales respecto de los cuales haya transmitido información previamente y que hayan estado pero que ya no estén en órbita terrestre.

42 Véase nota 41

43 En muchas ocasiones los Estados no suministran suficiente información sobre el objeto espacial que será lanzando al espacio ultraterrestre porque no quieren revelar la finalidad del satélite. Esto se puede evidenciar más que todo en los satélites lanzados con fines militares, más conocidos como "satélites espías". estar registrados o si se debe proporcionar información adicional en relación con los satélites inactivos, el fallo de misiones, los objetos espaciales que se han desprendido del satélite - desechos espaciales- y demás ocurrencias que hayan podido suceder en misiones espaciales. A la hora de establecer la responsabilidad, este tipo de información resulta de vital importancia para identificar el Estado responsable del daño ocasionado por el desecho espacial y para que el Estado al que se le causó un perjuicio pueda iniciar las reclamaciones pertinentes.

En materia de desechos espaciales resulta sumamente complejo identificar el Estado productor porque los desechos, en su mayoría, resultan irreconocibles tanto para el Estado productor del desecho como para el Estado perjudicado por el daño que este ocasionó y, por este mismo inconveniente, es altamente complicado iniciar una reclamación ante un Estado por los perjuicios ocasionados. La complejidad de la identificación de un desecho se puede originar por diferentes causas: i) si el daño sobreviene sobre un objeto espacial en el espacio ultraterrestre resulta demasiado complicado desplazarse hasta allí para determinar qué clase de desecho generó el daño. Asimismo, la rápida velocidad con que viajan los desechos espaciales (especialmente en LEO) incrementa las posibilidades de que estos perforen el satélite o la nave espacial y aun así continúen orbitando lo cual hace imposible encontrar el desecho; ii) cuando los objetos no se desintegran al ingresar a la atmósfera y caen a la superficie de la Tierra, por la fricción generada al entrar en contacto con la atmósfera los desechos cambian en muchos ca- 
sos sus condiciones físicas, hecho que dificulta aún más su identificación; iii) el pequeño tamaño de los desechos puede dificultar todavía más la identificación.

Otro artículo importante del Convenio de Registro es el VI, el cual determina que si un Estado Parte no logra identificar un objeto espacial que ha causado daño ${ }^{44}$ aplicando sus disposiciones, los otros Estados Partes, en especial aquellos que poseen instalaciones para la observación y rastreo de objetos espaciales, responderán a la solicitud formulada por el Estado parte que ha sufrido el daño para ayudar a identificar el objeto. En relación con este artículo, si bien es sabido que la buena fe es un principio internacional de derecho, puede ocurrir que un Estado que posee instalaciones de observación y rastreo, por intereses políticos no quiera colaborar con el Estado víctima del daño. Además, aunque el Tratado del Espacio, el Convenio sobre Responsabilidad y el Convenio de Registro fomentan la cooperación internacional, es posible que esta no suceda y por tanto el Estado que ha sufrido el daño no logre identificar el objeto que lo originó.

Otro punto débil de este Convenio es el artículo VII, el cual establece que el Convenio de Registro aplicará a las organizaciones internacionales gubernamentales que realicen actividades espaciales, siempre y cuando la organización acepte las obligaciones y derechos relacionados con el Convenio de Registro. Esta condición hace que el registro de objetos espaciales ante las Naciones Unidas no pueda ser completado,

44 El daño pudo haber sido ocasionado al Estado o alguna de sus personas físicas o morales o simplemente el objeto espacial puede ser de carácter peligroso o nocivo. porque algunos Estados son miembros de organizaciones de lanzamiento intergubernamentales pero no son parte del Convenio de Registro; con fortuna dichos Estados no contribuyen a la gran mayoría. Otro punto a tener en cuenta es que el Convenio no previó la admisión de compañías privadas tales como INTELSAT. ${ }^{45}$

Para la década de 1990, el registro de objetos espaciales decreció de manera sustancial ${ }^{46}$ alarmando profundamente a las Naciones Unidas y haciendo que la uncopuos implementara el artículo $\mathrm{X}^{47}$ del Convenio de Registro y bajo la dirección del Comité Jurídico expidiera la Resolución 59/115 de 2004 sobre la aplicación del Estado de lanzamiento, en la cual recomienda que Ios Estados que realizan actividades espaciales, en cumplimiento de los derechos y obligaciones internacionales que les concierne en virtud del Tratado del Espacio, del Convenio sobre Responsabilidad y del Convenio de Registro, promuevan en la legislación nacional la supervisión conti-

45 Benkö, M. and Kal-Uwe, S. (2005). Essential Air and Space Law Two. Eleven International Publishing, p. 36.

46

\begin{tabular}{|c|c|c|c|}
\hline Años & \multicolumn{3}{|c|}{ Número de objetos espaciales } \\
\hline & Lanzamientos & Registro & Porcentaje \\
\hline $1976-1980$ & 587 & 582 & $99 \%$ \\
\hline $1981-1985$ & 780 & 756 & $97 \%$ \\
\hline $1986-1990$ & 707 & 671 & $95 \%$ \\
\hline $1991-1995$ & 593 & 542 & $91 \%$ \\
\hline $1996-2000$ & 657 & 511 & $78 \%$ \\
\hline $2001-2003$ & 269 & 201 & $75 \%$ \\
\hline
\end{tabular}

47 Artículo $X$. Diez años después de la entrada en vigor del presente Convenio, se incluirá en el programa provisional de la Asamblea General de las Naciones Unidas la cuestión de un nuevo examen del Convenio, a fin de estudiar, habida cuenta de la anterior aplicación del Convenio, si es necesario revisarlo. No obstante, en cualquier momento una vez que el Convenio lleve cinco años en vigor, a petición de un tercio de los Estados Partes en el Convenio y con el sentimiento de la mayoría de ellos, habrá de reunirse una conferencia de los Estados Partes con miras a reexaminar este Convenio. Este nuevo examen tendrá en cuenta, en particular, todos los adelantos tecnológicos pertinentes, incluidos los relativos a la identificación de los objetos espaciales. 
nua de las actividades que llevan a cabo las entidades no gubernamentales que se encuentran bajo su jurisdicción, como también que se fomenten los acuerdos de lanzamiento conjuntos entre la empresa privada nacional y el Estado. ${ }^{48}$ Posteriormente, en el 2005, el uncopuos, con la ayuda del Comité Jurídico y del Comité Técnico y Científico, se dispuso a la tarea de estudiar una nueva serie de recomendaciones para que los Estados y organizaciones internacionales registraran los objetos espaciales, puesto que si bien el Convenio de Registro gozaba de aceptación universal en la comunidad internacional, con el paso del tiempo el uncopuos seguía notando con alarmante preocupación la rápida disminución de objetos registrados. Así, en el 2007, el Comité Jurídico a través de análisis hechos sobre el registro de objetos, adoptó por consenso la resolución sobre la práctica de los Estados y organizaciones internacionales en relación con el registro de objetos espaciales, la cual sería adoptada por consenso por la Asamblea General de la Naciones Unidas el 17 de diciembre de 2007, por medio de la Resolución 62/101 “Recomendaciones para mejorar las prácticas de los Estados y organizaciones internacionales en el registro de objetos espaciales" ${ }^{\prime 9}$. En ella se recomienda a los Estados aumentar los registros de objetos espaciales de las organizaciones intergubernamentales internacionales según el artículo III del Convenio de Registro e invita a las legislaciones que no han adoptado el Con-

48 Documentos Oficiales de la Asamblea General, quincuagésimo cuarto período de sesiones, Suplemento $N^{\circ} 20$ y corrección (A/54/20 y Corr.1).

49 Benkö, M. and KAI-Uwe, (2005). Essential Air and Space Law Two. Eleven International Publishing, p. 7. venio sobre el Registro a que lo hagan; mejorar sus procedimientos para el reconocimiento de objetos espaciales tal como consta en el artículo VI del Convenio de Registro; que los Estados Partes en el Convenio sobre el Registro y las organizaciones gubernamentales internacionales que lleven a cabo actividades espaciales, una vez hayan adoptado los derechos y obligaciones en virtud del Convenio proporcionen toda la información, establezcan un registro apropiado y lo notifiquen al Secretario General de las Naciones Unidas; que aquellas organizaciones gubernamentales internacionales que no hayan aceptado los derechos y obligaciones del Convenio de Responsabilidad inicien a hacerlo de conformidad con el artículo VII; que se estudie la posibilidad de lograr la uniformidad en el tipo de información que se suministra al Secretario General de las Naciones Unidas a la hora de registrar un objeto espacial, entre otras.

No obstante todas estas recomendaciones aprobadas por Naciones Unidas, el resultado no ha sido el esperado a pesar de que, como se puede evidenciar, el Estado de registro es fundamental para la seguridad y el manejo del tráfico espacial pues constituye, no solo una medida de "construcción de confianza" en relación con aspectos de seguridad tanto nacional como internacional, sino una demostración de la transparencia y honestidad hacia la comunidad internacional; 50 además, es un elemento primordial para determinar la responsabilidad dentro del Convenio sobre Responsabilidad y el Convenio de Registro. Sin embargo, las normas contenidas en los

50 Benko, M. (2005). Essential Air and Space Law. Eleven International Publishing, p. 8. 
tratados no proveen información suficiente para solucionar eventuales daños causados por desechos espaciales, debido a la falta de precisión en los términos y de soluciones efectivas para indemnizar los perjuicios.

\section{Desechos espaciales y el Convenio so- bre la responsabilidad internacional por daños causados por objetos espaciales}

En el Convenio sobre la Responsabilidad los Estados reconocieron la necesidad de elaborar normas y procedimientos internacionales eficaces sobre la responsabilidad por daños causados por objetos espaciales, en particular, de “asegurar el pago rápido (...) de una indemnización plena y equitativa a las víctimas de tales daños" ${ }^{51}$ e instauraron una reglamentación más específica definiendo conceptos establecidos en el Tratado del Espacio, tales como daño ${ }^{52}$, lanzamiento ${ }^{53}$, estado de lanzamiento ${ }^{54}$, objeto

51 Preámbulo del Convenio sobre la responsabilidad internacional por daños causados por objetos espaciales, aprobado por la Asamblea General en su Resolución 2777 (XXVI), el 29 de noviembre de 1971 "Reconociendo la necesidad de elaborar normas y procedimientos internacionales eficaces sobre la responsabilidad por daños causados por objetos espaciales y, en particular, de asegurar el pago rápido, con arreglo a lo dispuesto en el presente Convenio, de una indemnización plena y equitativa a las víctimas de tales daños".

52 Artículo I literal a). Se entenderá por "daño" la pérdida de vidas humanas, las lesiones corporales u otros perjuicios a la salud, así como la pérdida de bienes o los perjuicios causados a bienes de Estados o de personas físicas o morales, o de organizaciones internacionales intergubernamentales.

53 Artículo I literal b). El término "lanzamiento" denotará también todo intento de lanzamiento.

54 Artículo I literal c). Se entenderá por "Estado de lanzamiento": i) Un Estado que lance o promueva el lanzamiento de un objeto espacial; ii) Un Estado desde cuyo territorio o desde cuyas instalaciones se lance un objeto espacial. espacial $^{55}$ y régimen de responsabilidad ${ }^{56}$. El enfoque que tuvo el Convenio sobre Responsabilidad fue crear reglas y procedimientos para las actividades realizadas en el espacio ultraterrestre por los Estados miembros, en caso de que ocurriera un daño que ocasionara la pérdida de vidas humanas, lesiones corporales u otros perjuicios a la salud (lesiones físicas o morales), pérdida de bienes, perjuicios causados a bienes de Estados u organizaciones internacionales o intergubernamentales. ${ }^{57}$ Sin embargo, no es posible hablar de los desechos espaciales y la responsabilidad aplicable a estos, sin antes ocuparse de la responsabilidad en general y de la responsabilidad internacional.

\section{Responsabilidad civil}

El derecho del espacio ultraterrestre, a pesar de ser una rama con diferentes exigencias a las demás, establece que los elementos esenciales para que haya lugar a responsabilidad son: el hecho generador, el daño y el nexo causal entre ambos. Igualmente, la categoría de respon-

55 Artículo I literal d). El término "objeto espacial" denotará también las partes componentes de un objeto espacial, así como el vehículo propulsor y sus partes.

56 Artículo II y III.

57 Artículo I. "A los efectos del presente Convenio: a) Se entenderá por "daño" la pérdida de vidas humanas, las lesiones corporales u otros perjuicios a la salud, así como la pérdida de bienes o los perjuicios causados a bienes de Estados o de personas físicas o morales, o de organizaciones internacionales intergubernamentales; b) El término "lanzamiento" denotará también todo intento de lanzamiento; c) Se entenderá por "Estado de lanzamiento": i) Un Estado que lance o promueva el lanzamiento de un objeto espacial; ii) Un Estado desde cuyo territorio o desde cuyas instalaciones se lance un objeto espacial; d) El término "objeto espacial" denotará también las partes componentes de un objeto espacial, así como el vehículo propulsor y sus partes." 
sabilidad que surge bajo este régimen es el de la responsabilidad extracontractual, en cuanto no existe un vínculo previo entre la víctima y el autor del daño. Adicionalmente, los tipos de atribución de responsabilidad aplicables son el objetivo y el subjetivo, dependiendo de las circunstancias en las cuales se haya generado el daño. En el sistema de responsabilidad subjetivo, el Estado que reclame la responsabilidad de otro por sus actividades espaciales tiene la carga de la prueba, es decir, está en la obligación de probar como mínimo que el Estado demandado ha actuado negligentemente. Esto favorece al Estado que generó el daño puesto que la carga de la prueba está en cabeza del afectado, al cual le quedará supremamente complicado probar la culpa del otro Estado. ${ }^{58}$ Por otro lado, en el sistema de responsabilidad objetiva o absoluta, como es comúnmente conocido en otras legislaciones, la víctima no tiene que probar culpa o negligencia por parte del Estado cuyo objeto espacial causó el daño, por lo que solo sería necesario establecer una relación de causalidad entre el objeto espacial y el daño producido por este. En cuanto al derecho del espacio ultraterrestre, el tipo de responsabilidad que este utiliza es un sistema de responsabilidad dual, "según el cual el régimen de responsabilidad por daños causados por un objeto espacial o sus partes componentes se determina en la aplicación del principio de la responsabilidad absolu-

58 Dembling, P.G. Establishing Liability for Outer Space Activities. En: Space Law perspectives. Schwartz, p. 229. "si la víctima de un accidente espacial tuviera que probar culpa o negligencia, sus posibilidades reales de obtener compensación por los daños sufridos serían virtualmente inexistentes". ta en ciertos casos, y el principio de la responsabilidad por culpa, en otros." 59

\section{Responsabilidad internacional de los Estados}

Por otro lado, el derecho del espacio ultraterrestre es una rama autónoma del derecho internacional, el cual establece que los Estados son los protagonistas del derecho internacional y por tanto son sujetos de derechos y obligaciones bajo el principio fundamental de soberanía. ${ }^{60}$ Es de anotar que el derecho espacial se complementa por mandato del Tratado del Espacio y forman parte de él, igualmente, el derecho internacional y las reglamentaciones de las Naciones Unidas. ${ }^{61}$ En efecto, es importante tener en cuenta que para que haya lugar a la responsabilidad internacional es necesario que se haya quebrantado una norma de derecho internacional; así quedó consignado cuando las Naciones Unidas aprobó el Tratado sobre los principios que deben regir las actividades de los Estados en la exploración y utilización del espacio ultraterrestre, incluso la Luna y otros cuerpos celestes (Tratado del Espacio), el 19 de diciembre de 1966, aprobado por la Asamblea General mediante la Resolución 2222 (XXI) en 1967, en el cual se declaró que los Estados realizarían acti-

59 MARChÁN, J. (1987). Derecho internacional del espacio. Banco Central del Ecuador, p. 588.

60 BRotóns, R. (2007). Derecho internacional. Canadá McGraw-Hill, p. 41.

61 Tratado del Espacio, artículo III. Los Estados Partes en el Tratado deberán realizar sus actividades de exploración y utilización del espacio ultraterrestre, incluso la Luna y otros cuerpos celestes, de conformidad con el derecho internacional, incluida la Carta de las Naciones Unidas, en interés del mantenimiento de la paz y la seguridad internacionales y del fomento de la cooperación y la comprensión internacionales. 
vidades de exploración y uso del espacio ultraterrestre y también de todos los cuerpos celestes "de acuerdo con el derecho internacional, incluida la Carta de las Naciones Unidas"62.

\section{Responsabilidad espacial}

Si bien la actividad espacial trae a la humanidad un sinfín de beneficios, no puede ignorarse el hecho de que esta pone en peligro a muchos individuos y Estados, además de los que se arriesgan directamente a sufrir daños o pérdidas por la participación en ellas. No en vano el Tratado del Espacio reconoce que "los experimentos peligrosos desarrollados en el espacio ultraterrestre pueden afectar incluso a toda la humanidad, cambiar el medio ambiente de la Tierra, contaminar la atmósfera y producir efectos incalculables sobre la vida"63 y que "los vehículos espaciales o partes de los cohetes que pueden estrellarse en la Tierra y causar graves daños a la vida y a la propiedad, también pueden chocar con aviones $u$ otros objetos voladores" 640 producir interferencia entre los satélites. En razón a esto, el derecho espacial además de regir los derechos y las obligaciones de los Estados en esta materia, debió extenderse también al ámbito de la responsabilidad, por lo cual la comunidad internacional por medio de las $\mathrm{Na}$ -

62 Artículo III del Tratado de los principios que deben regir las actividades de los Estados en la exploración y uso del espacio ultraterrestre, incluida la Luna y otros cuerpos celestes. Aprobado por la Asamblea General de las Naciones Unidas el 19 de diciembre de 1966. Naciones Unidas, Oficina de Asuntos del Espacio Ultraterrestre, Tratados y principios de las Naciones Unidas sobre el espacio ultraterrestre y resoluciones conexas de la Asamblea General. Nueva York, 2008.

63 LACHs, M. (1977). El derecho del espacio ultraterrestre. Buenos Aires: Fondo de Cultura Económica, p. 159.

64 Ibid., p.160. ciones Unidas crea el Convenio sobre la responsabilidad internacional por daños causados por objetos espaciales, aprobado por la Asamblea General en su Resolución 2777 (XXVI), el 29 de noviembre de 1971.

\section{Responsabilidad espacial objetiva}

Como se mencionó anteriormente, el derecho espacial tiene una responsabilidad dual y esta se evidencia en el Convenio sobre Responsabilidad, puesto que el artículo II $^{65}$ establece el régimen de la responsabilidad objetiva cuando i) Ios daños se produzcan en la superficie terrestre y ii) cuando los daños son causados a una aeronave en vuelo. En este régimen "el demandante no tiene la necesidad de probar que el daño causado por la conducta del demandado se debe a una acción u omisión dolosa o negligente por parte del mismo"66. Cuando se aplica el principio de la responsabilidad objetiva se pretende brindar una mayor protección a las víctimas de los daños que puedan causar los objetos espaciales, en especial a los países en vía de desarrollo, puesto que estos tienen un papel pasivo frente a la mayoría de actividades espaciales y sin una adecuada protección jurídica se encontrarían en una posición desventajosa para asumir los daños y los costos de un accidente espacial. ${ }^{67}$ De este modo, si ocurre un daño amparado por el artículo II del Convenio, el Estado

65 Un Estado de lanzamiento tendrá responsabilidad absoluta y responderá de los daños causados por un objeto espacial suyo en la superficie de la Tierra o a las aeronaves en vuelo.

66 GutiéRrez Espada, C. (1979). La responsabilidad internacional por daños en el derecho del espacio, Secretaria de Publicaciones Universidad de Murcia. España, p. 59.

67 MARCHÁN. Op. cit., p. 593. 
lesionado tiene que demostrar únicamente la existencia de una relación causa efecto.

El hecho que el régimen de responsabilidad objetiva aplique a los daños causados en la Tierra por un objeto espacial tiene una doble justificación. En primer lugar, se debe a que la actividad espacial es una actividad riesgosa en la que: i) el objeto espacial tiene una posición ventajosa respecto a los terceros en tierra; ii) existe en la mayoría de los casos una imposibilidad por parte de la víctima de obtener prueba de la culpa del operador espacial y iii) el uso de aparatos creadores de riesgos para los terceros genera para el operador la obligación de responder frente a las personas que nada tienen que ver con el uso de dichos aparatos. ${ }^{68}$ En segundo lugar, nace de la necesidad de reparar el perjuicio y de concederles una pronta indemnización plena y equitativa a las víctimas de los daños ${ }^{69}$, al ser estos terceros quienes no obtienen ningún provecho de los riesgos de la actividad espacial.

Por otro lado, si bien el Convenio sobre Responsabilidad no estableció qué tipo de relación de causalidad (nexo causal) era aplicable para poder determinar la responsabilidad, de la normativa del Convenio se desprende que al aplicarse el principio de responsabilidad objetiva, lo importarte debe ser encontrar al Estado en cuyo

68 Melon, G. (1967). International Liability for Space Activity. En: Proceedings of the Tenth Colloquium on the Law of Outer Space. Belgrado, p. 185.

69 Preámbulo del Convenio sobre responsabilidad por daños causados por objetos espaciales: "Reconociendo la necesidad de elaborar normas y procedimientos internacionales eficaces sobre la responsabilidad por daños causados por objetos espaciales $y$, en particular, de asegurar el pago rápido, con arreglo a lo dispuesto en el presente Convenio, de una indemnización plena y equitativa a las víctimas de tales daños". registro aparece el objeto causante del daño. Así, el Estado que aparece en el registro como propietario del objeto, teniendo la jurisdicción sobre este, se denomina Estado de lanzamiento de dicho objeto, el cual ha sido definido en el literal c) del artículo I del Convenio ${ }^{70}$ como: i) un Estado que lance o promueva el lanzamiento de un objeto espacial o ii) un Estado desde cuyo territorio o desde cuyas instalaciones se lance un objeto espacial. Por lo tanto, puede decirse respecto al nexo causal, que el Estado de lanzamiento del objeto causante del daño es el sujeto internacionalmente responsable de pagar la indemnización a las víctimas.

\section{Responsabilidad espacial subjetiva}

El Convenio de Responsabilidad establece que cuando los daños son causados a un objeto espacial o a bienes a bordo del mismo o a su tripulación, el Estado de lanzamiento que cause el daño, bien sea sobre la superficie de la tierra o en el espacio ultraterrestre, será responsable si el daño se produjo por su culpa (artículo III) ${ }^{71}$. De lo anterior se puede deducir que la responsabilidad aplicable en estos casos es

70 Artículo I literal c). "Se entenderá por 'Estado de lanzamiento': i) un Estado que lance o promueva el lanzamiento de un objeto espacial; ii) un Estado desde cuyo territorio o desde cuyas instalaciones se lance un objeto espacial".

71

Convenio sobre la responsabilidad por daños causados por objetos espaciales. Artículo III: "cuando el daño sufrido de la superficie de la Tierra por un objeto espacial de un Estado de lanzamiento, o por las personas o los bienes a bordo de dicho objeto espacial, sea causado por un objeto espacial de otro Estado de lanzamiento, este último Estado será responsable únicamente cuando los daños se hayan producido por su culpa o por culpa de las personas de que sea responsable" y artículo IV inciso 1, literal b: "Si los daños han sido causados a un objeto espacial de un tercer Estado, o a las personas o los bienes a bordo de ese objeto espacial, fuera de la superficie de la Tierra, la responsabilidad ante ese tercer Estado se fundará en la culpa de cualquiera de los dos primeros Estados o en la culpa de las personas de que sea responsable cualquiera de ellos". 
la responsabilidad subjetiva, en cuanto no solo debe probarse el daño causado y el nexo causal entre este y su origen, sino que también debe probarse la culpa. En efecto, en caso tal de que no se logre probar la culpa, no hay obligación de reparar el perjuicio y la carga de la prueba se encuentra en manos de quien busca ser indemnizado. Como antecedente histórico vale anotar que algunos países se opusieron a lo estipulado en el artículo III durante la negociación del Convenio, pues consideraban que tratar el tema de la colisión de objetos espaciales era una cuestión precipitada. Además, algunos Estados estimaban que el Convenio debía abordar solo los daños causados a países no participantes en las actividades espaciales. No obstante, en la negociación se llegó a la conclusión que aplicar el principio de la responsabilidad absoluta en el caso de accidente entre dos objetos espaciales, llevaría al absurdo de que los Estados deberían compensarse recíprocamente por los daños que se causasen entre sí: "si ambos Estados participan en actividades espaciales, no se da el caso de desigualdad entre las partes: no existe la víctima indefensa y sin recursos versus la potencia espacial; ambos Estados conocen la tecnología aplicable y ambos conocen y han aceptado los riesgos"72. Por tanto, el artículo III limita la aplicación del principio de responsabilidad absoluta, determinando que cuando el daño se produzca fuera de la superficie de la Tierra, al Estado autor del daño le será imputada la responsabilidad únicamente cuando el daño se haya producido por su culpa.

72 Matte, N. (1979). Aerospace and Space Law. Annals of Air and Space Law. Institute and Centre of Air and Space Law, McGill University, Montreal, Vol. IV, p. 161.

\section{Análisis de la responsabilidad espacial y los desechos espaciales}

En relación con la aplicación del Convenio de Responsabilidad y los desechos espaciales, algunos doctrinantes argumentan que resultaría de gran ayuda para resolver el problema que producen los desechos espaciales cuando estos chocan con otro objeto espacial, es decir, para determinar el tipo de responsabilidad por el daño causado por dichos desechos sobre otros objetos espaciales. ${ }^{73}$ Esta interpretación del Convenio se debe a que, tal como lo expone el artículo I literal d), "el término "objeto espacial" denotará también las partes componentes de un objeto espacial, así como el vehículo propulsor y sus partes", lo cual permite que en dicha definición los desechos espaciales encajen perfectamente.

Sin embargo, bajo el supuesto que los desechos espaciales pueden estar incluidos en el término “objeto espacial", surgen alrededor del Convenio sobre Responsabilidad varias incógnitas: i) ¿qué tipo de responsabilidad le es aplicable a un desecho espacial que cause daños en la superficie de la Tierra? Bajo el Convenio de Responsabilidad, el régimen aplicable a los desechos caídos en la superficie de la Tierra sería la responsabilidad objetiva. Sin embargo, si el desecho no resulta ser identificado, bien sea por su tamaño o por el cambio físico que tuvo por la fricción al ingresar a la atmósfera, sería sumamente difícil identificar el Estado responsable del desecho

\footnotetext{
73 Hobe, S. and Mey, J. H. (2009). Space low and space debris. International Interdisciplinary Congress on Space Debris. May 7 to 9. McGill University, Institute of Air and Space Law. Montreal, Canada, p. 401.
} 
(Estado responsable del daño) porque este no resulta reconocible, y por tanto el daño causado a la superficie de la Tierra o persona perjudicada no podría ser indemnizada porque no se encuentra identificado el Estado de lanzamiento o el Estado de registro. Aquí se evidencia lo peligrosa que resulta la actividad espacial, puesto que i) el desecho espacial tiene una posición ventajosa respecto a los terceros en tierra; ii) existe en la mayoría de los casos una imposibilidad por parte de la víctima de obtener prueba de la culpa del operador espacial; y iii) el uso de aparatos creadores de riesgos (desecho espacial) para los terceros genera para el operador la obligación de responder frente a las personas que nada tienen que ver con el uso de dichos aparatos ${ }^{74}$. Es clara aquí la necesidad, dentro de la comunidad internacional, de crear una normatividad específica para los desechos espaciales y la responsabilidad derivada de estos; ii) ¿qué tipo de responsabilidad le es aplicable a un desecho espacial que cause daños en el espacio ultraterrestre? Bajo el Convenio de Responsabilidad, el tipo de responsabilidad aplicable a los desechos en el espacio ultraterrestre sería la responsabilidad subjetiva. Por tanto, la víctima debe probar el daño causado, el nexo causal entre este y su origen, y adicionalmente la culpa. En caso tal que no se logre probar la culpa o no se pueda identificar el objeto que cause el daño, no hay obligación de reparar el perjuicio, ya que la carga de la prueba se encuentra en manos de quien busca ser indemnizado y esto resultaría sumamente complejo para la víctima puesto que los desechos en muchas ocasiones, por su tamaño, son de difícil

74 Meloni. Op. cit., p. 185. identificación. Aun más, probar la culpa resultaría ser una labor casi imposible pues un desecho es un objeto que se encuentra a la deriva en el espacio ultraterrestre, no puede ser maniobrado $y$, por tanto, no es clara la intencionalidad o negligencia con que actúa el Estado generador de desechos. Otro punto a tener en cuenta es que el Convenio de Responsabilidad no se refirió a la culpa de manera directa, razón por la cual si bien el Convenio en el artículo II determina la responsabilidad absoluta del Estado de lanzamiento por los objetos espaciales que causen daño a otro objeto de un Estado, sus nacionales $\mathrm{u}$ organizaciones internacionales, no establece cuál ha de ser el trato de la negligencia o culpa para daños causados en el espacio ultraterrestre de manera específica y concreta sino más bien interpretativa (artículos III y IV inciso 1, literal b). Un argumento en contra de esta posición resulta ser que el derecho debe ser interpretado de manera armónica y por lo tanto al no especificarse qué tipo de responsabilidad le es aplicable de manera específica y directa, deben ser las normas generales de la responsabilidad las que deben llenar el "vacío" legislativo del Convenio sobre Responsabilidad. Sin embargo, al no definir el Convenio de Responsabilidad la culpa en sí y tampoco establecer con precisión el parámetro con el cual se debe determinar la negligencia, aun así se apliquen las normas generales de la Responsabilidad, debido a la magnitud de los desechos espaciales y el papel tan importante que juegan actualmente en el derecho espacial, es necesaria una normatividad específica y concreta que reglamente la responsabilidad sobre los desechos espaciales para tener claridad al respecto; iii) ¿cómo se determina la identidad de 
Ios desechos espaciales? El Convenio sobre Responsabilidad no estipuló un mecanismo específico para determinar la identidad de los objetos espaciales y menos aún para identificar los desechos espaciales que se derivan del lanzamiento de un objeto espacial al espacio ultraterrestre. Por tal razón, resulta sumamente complicado que cuando un desecho produzca daños bien sea a una persona, a la superficie de la Tierra o a un objeto espacial, por ejemplo un satélite, se determine de manera eficaz el responsable del daño y, en particular, se asegure el pago rápido y una indemnización plena y equitativa a las víctimas de tales daños, por la dificultad que existe en determinar quién fue el productor del desecho espacial; no puede haber indemnización sin antes haber identificado al infractor que causó el daño ${ }^{75}$. Dicho Convenio opera bajo el supuesto que el "Estado de lanzamiento tendrá responsabilidad absoluta y responderá por daños causados por un objeto espacial suyo en la superficie de la Tierra o a las aeronaves en vuelo", lo cual no resulta suficiente para abordar y solucionar futuros accidentes relacionados con desechos espaciales; iv) El Convenio sobre Responsabilidad habla simplemente sobre daños causados a personas o bienes, pero ¿qué ocurre con los daños causados al medio ambiente o a cualquier otro bien común en el espacio ultraterrestre por la gran cantidad de desechos en el espacio? Dicho Convenio no hace referencia específica sobre los daños al medio ambiente y mucho menos qué tipo de responsabilidad se deriva por la ocurrencia de estos daños. Resulta, por consiguiente, sumamente importante que la comunidad

75 DiederiKs-VerschoOR, I.H. y Kopal, V. Op. cit., p. 125 internacional produzca una normatividad específica en relación con los desechos espaciales, puesto que de no hacerlo se estaría violando de manera directa el Tratado del Espacio ${ }^{76}$ que establece en el artículo IX, que los Estados Partes del Tratado "harán los estudios e investigaciones del espacio ultraterrestre, incluso la Luna y otros cuerpos celestes, y procederán a su exploración de tal forma que no se produzca una contaminación nociva ni cambios desfavorables en el medio ambiente de la Tierra" (resaltado fuera del texto original). Actualmente el espacio ultraterrestre tiene una alta cantidad de desechos que han venido creciendo de manera exponencial, lo cual ha producido contaminación nociva para las actividades espaciales y fenómenos desfavorables que eventualmente afectarán a la Tierra si no se toman medidas necesarias para determinar la responsabilidad por daños ocasionados por desechos espaciales y su mitigación. A manera de ejemplo, en 1978 la Unión Soviética argumentó que los daños al medio ambiente no estaban incluidos en el Convenio de Responsabilidad, cuando su satélite Cosmos 954 que contenía un reactor nuclear con materiales altamente radioactivos (20 kgm de uranio-235), cayó en Canadá generando 50 fragmentos de desechos y más de 4.000 partículas con material radioactivo, las cuales se esparcieron en 124.000 km² $^{2}$ sobre Alberta y Saskatchewan, generando daños en el medio ambiente. Debido a la gravedad del problema y la urgencia con que se debía actuar para solucionarlo, Canadá, con la colaboración de Estados Unidos, realizó las la-

76 Es relevante resaltar que la Convención de Responsabilidad debe ser interpretada de manera armónica con el Tratado del Espacio tal y como se expresa en su preámbulo. 
bores de limpieza conocidas como Operación luz de la mañana (Operation Morning Light) y logró recuperar alrededor del $0.1 \%$ de la energía nuclear, con un costo de alrededor de 14 millones de dólares canadienses. ${ }^{77}$ Por lo ocurrido, Canadá presentó una reclamación ante las Naciones Unidas, siendo esta la primera exigencia y tal vez la única generada bajo el Convenio sobre la responsabilidad internacional por daños causados por objetos espaciales y bajo principios de derecho internacional hasta el momento. En dicha reclamación Canadá probó que el objeto espacial fue lanzado por la Unión Soviética; no obstante la Unión Soviética la refutó argumentando que este Estado había realizado operaciones de limpieza innecesarias, no le había sido permitido ayudar a realizar la limpieza ${ }^{78}$, y que en la definición del término "daño" el Convenio sobre Responsabilidad no había incluido el daño al medio ambiente. Finalmente, en 1981 la Unión Soviética llegó a un acuerdo con Canadá y le pagó la suma de 3 millones de dólares canadienses.

De otra parte, el Convenio de Responsabilidad se queda corto al tratar de abordar el crecimiento de la actividad espacial, puesto que la cantidad de actores dentro del espacio ultraterrestre ha venido aumentando y se hace cada vez más

77 KovudhiKulrungsri, L. y NAKSEEHARACH, D. (2011). Liability Regime of International Space Law: Some Lessons from International Nuclear Law. Leiden University. The Netherlands, p. 6: "in collaboration with the United States, Canada set the clean-up operation, Operation Morning Light which finished in October 1978 and resulted in an estimated recovery about 0.1 percent of Cosmos 954 nuclear power source. 22 This operation cost approximately CAD 14 million".

78 La URSS, basándose en el Acuerdo sobre el salvamento y devolución de astronautas de 1968, el cual estipula que al Estado que ha causado los daños debe dársele la oportunidad de ayudar en la búsqueda y rescate de la operación, para así facilitar la reducción de los daños (artículos 5 y 2 del Acuerdo). Sin embargo, Canadá se negó a recibir la ayuda de URSS. DiederIKs-Verschoor, I. H. Op. cit., Philepina, p. 42. notoria la privatización del espacio ultraterrestre. ${ }^{79}$ En efecto, si bien los Estados son responsables por las actividades espaciales de los particulares bajo su jurisdicción ${ }^{80}$, la industria espacial privada se ha venido incrementando sustancialmente debido a que las legislaciones nacionales han permitido el desarrollo del mercado de las telecomunicaciones, haciendo que más industrias nacionales y tratados comerciales entre naciones estén involucrados con las actividades espaciales, para lo cual no existe una reglamentación definitiva a la cual estas organizaciones puedan adherirse frente a una futura responsabilidad por daños y problemas con los desechos espaciales.

\section{CONCLUSIONES}

Bajo el análisis previamente planteado, los tratados multilaterales que forman parte del núcleo de derecho del espacio ultraterrestre proveen,

79 El gran desarrollo de la tecnología espacial y los beneficios que esta le ha aportado a la sociedad ha hecho que actualmente, tanto el Estado como las empresas privadas realicen actividades espaciales, por lo cual, ya no solo se habla de las agencias espaciales de cada Estado sino también de agencias espaciales privadas tales como Spaceport America, Virgin Galactic, SpaceX e Intelsat (International TelecommunicationsSatellite Organization), luego de su privatización en el 2001. Recuperado el 14 de mayo de 2012, en: http://space.about.com/od/ privatespaceagencies/Private_and_Commercial_Space_Exploration_ Agencies.htm

80 Tratado del Espacio, artículo VI. Los Estados Partes en el Tratado serán responsables internacionalmente de las actividades nacionales que realicen en el espacio ultraterrestre, incluso la Luna y otros cuerpos celestes, los organismos gubernamentales o las entidades no gubernamentales, y deberán asegurar que dichas actividades se efectúen en conformidad con las disposiciones del presente Tratado. Las actividades de las entidades no gubernamentales en el espacio ultraterrestre, incluso la Luna y otros cuerpos celestes, deberán ser autorizadas y fiscalizadas constantemente por el pertinente Estado Parte en el Tratado. Cuando se trate de actividades que realiza en el espacio ultraterrestre, incluso la Luna y otros cuerpos celestes, una organización internacional, la responsabilidad en cuanto al presente Tratado corresponderá a esa organización internacional y a los Estados Partes en el Tratado que pertenecen a ella. 
hasta cierto punto, reglas, procedimientos y mecanismos legales los cuales podrían ser usados en el futuro para solucionar los problemas generados por la proliferación de desechos espaciales. Sin embargo, por más ampliamente que se interpreten las normas, desde una perspectiva crítica y contextualizada sobre la gravedad del problema el texto de los tratados se caracteriza por la falta de términos adecuados para la regulación de desechos espaciales, como también de procedimientos y mecanismos para solucionar posibles disputas que se puedan originar por daños.

Igualmente, existen dos posiciones interpretativas frente a los convenios analizados (Tratado del Espacio, Convenio sobre Responsabilidad y Convenio de Registro) sobre daños causados por objetos espaciales. La primera posición establece que, si bien los convenios no mencionan de manera explícita los desechos espaciales dentro de su definición de "objeto espacial”, estos pueden ser incluidos y la responsabilidad que es aplicable a los objetos espaciales también puede serlo a los daños que causen los desechos espaciales. Sin embargo, desde un punto de vista crítico, el hecho que los desechos espaciales se incluyan en esta definición constituye simplemente un medio para disuadir a los Estados para que los aminoren pero no soluciona el problema a profundidad, porque no hay respuesta clara para solucionar un eventual problema, entre otras cosas porque técnicamente presenta una serie de dificultades que son de naturaleza evidente. En concordancia con esta crítica, la segunda posición arguye que el término "objeto espacial" no incluye los desechos espaciales dentro de los convenios, por lo cual resulta necesaria la creación de una reglamentación jurídica (tratado) en la cual se establezca un régimen específico de responsabilidad aplicable a los desechos, ya que esto ayudaría a evitar futuras ambigüedades a la hora de resolver e indemnizar los daños ocasionados por estos. El único instrumento jurídico que podríamos encontrar en la normatividad espacial, que intenta regular la materia de manera tímida, es la Resolución 62/217 de 2007 del Comité de Usos Pacíficos del Espacio Ultraterrestre.

Más aún, desde el punto de vista del derecho espacial, para que haya lugar a la responsabilidad es necesario que se viole una de sus normas, por lo tanto, el hecho de que no exista una norma en este ámbito que regule la materia sobre desechos espaciales, hace que no pueda haber violación y, por consiguiente, tampoco haya lugar a la responsabilidad ${ }^{81}$. Si bien es cierto que el Comité Técnico y Científico adoptó en el 2007, de manera consensual, parámetros para la mitigación de la proliferación de desechos espaciales, y que dichos parámetros fueron subsecuentemente adoptados por el comité principal de uncopuos el 15 de junio de 2007, apoyado por la Asamblea General de las Naciones Unidas por consenso en la Resolución 61/111 sobre "Cooperación internacional en los usos pacíficos del espacio ultraterrestre", en febrero 15 de 2008, es necesario que el Comité Jurídico expida normas que sean reconocidas por la comunidad internacional por su carácter vinculante y no simplemente como recomenda-

81 KARRESt, A. (2009). Liability for Damages Cause by Space Activities. The development of outer space: sovereignty and property rights in international space law. Santa Barbara, Praeger, p. 108. 
ciones. Así, el derecho espacial ultraterrestre debe arreglar esta laguna jurídica y por medio de la comunidad internacional llenar el vacío con una normatividad que implemente términos adecuados, procedimientos y mecanismos, tanto de mitigación de desechos, como de solución de disputas que se generen por la responsabilidad por los daños ocasionados por desechos espaciales tanto en la Tierra como en el espacio ultraterrestre, debido a la proliferación del uso del espacio por la sociedad actual. Todavía más, la regulación que surja en esta materia debe agravar la responsabilidad cuando los daños hayan sido producidos por objetos o desechos que contengan o hayan contenido carga nuclear.

\section{Bibliografía}

Andrenucci, M., Pergola, P., Ruggiero, A. Active Removal of Space Debris, Expanding foam application for active debris removal, final report, 2011.

BAKER, Howard A. (1989). Space Debris: Legal and Policy Implications. University of Colorado Law Review 55.

Benkö, Marietta and KAI-UwE, Schrogl. (2005). Essential Air and Space Law Two. Eleven International Publishing.

Benkö, Marietta. (2005). Space Law: Current Problems and Perspectives for Future ReguIation. Utrecht, The Netherlands: Eleven International Pub.

BEnkö, Marietta and KAI-Uwe,Schrogl.(2008). European Space Policy Institute.German Journal of Air and Space Law 335.
BRotóns, Ramiro. (2007). Derecho Internacional. McGraw-Hill.

Dembling, P.G. Establishing Liability for Outer Space Activities. En: Space Law perspectives. Schwartz.

Diederiks-Verschoor, I.H. y Kopal, V. (2008). An Introduction to Space Law. Kluwer Law International. The Netherlands. Third Revised Edition.

Ferguson, Katty. (2000). La medida del universo: la histórica búsqueda de maneras para cuantificar el espacio. Ed. Robinbook. Barcelona, España.

GutiéRrez EspadA, C. (1979). La responsabilidad internacional por daños en el derecho del espacio. Secretaría de Publicaciones Universidad de Murcia. España.

Gangale, Thomas. (2009). The Development of Outer Space: Sovereignty and Property rights in international space law. Santa Barbara, Praeger.

Gıorgı, Abetti. (1956). Historia de la Astronomía. Fondo de Cultura Económica. México.

Grossman, Karl. (1997). The Wrong Stuff: The Space Program's Nuclear. Common Courage Press. Atlanta Book Company. Common Courage Press, p. 45.

LofT, Kurt. (1999). Too Close for Comfort? The Tampa Tribune Press.

Hobe, Stephan and Mey, Jan Helge. Space low and space debris. International Interdiscipli- 
nary Congress on Space Debris. May 7 to 9, 2009. McGill University, Institute of Air and Space Law. Montreal, Canada.

Jasentuliyana, Nandasari. (1998). Space Debris and International Law. 29 Journal of Space Law, V. 139.

Johnson, Nicholas L., Stansbery, Eugene, Whitlock, David O., Abercromby, Kira J., Shoots, Debra. 2007. History of On-Orbit Satellite Fragmentations. Orbit Debris Program Office. 14th Edition. Texas, USA.

KovUdhIKULRUnGsRI, Lalin y NaKseeharaCh, Duangden. (2011). Liability Regime of International Space Law: Some Lessons from International Nuclear Law. Leiden University. The Netherlands.

KarRest, Armel. (2009). Liability for Damages Cause by Space Activities. The development of outer space: sovereignty and property rights in international space law. Santa Barbara, Praeger.

Kessler, Donald. (1978). Collision Frequency of Artificial Satellites: The Creation of a Debris Belt. Journal of Geophysical Research, Vol. 83, No. A6.

McAvor, Joseph J. (2004). Nuclear Space and the Earth Enviroment: the Benefits, Dangers and Legality of Nuclear Power and Propulsion in Outer Space. William and Mary Environmental Law and Policy Review, Vol. 29.

MARCHÁN, Jaime. (1987). Derecho internacional del espacio. Banco Central del Ecuador.
Meloni, Giovanni. (1967). International Liability for Space Activity. Proceedings of the Tenth CoIloquium on the Law of Outer Space. Belgrado.

Matte, Nicolas. (1979). Aerospace and Space Law. Annals of Air and Space Law. Institute and Centre of Air and Space Law, McGill University, Montreal, Vol. IV.

Zinner, N., Williamson, A., Brenner, K., Curran, J.B., Isaak, A., Knoch, M., Leppek, A., Lestishen, J., HunTER, J. Autonomous Rendezvous, Capture, and De-Orbit of Orbital Debris. Revolutionary Aerospace Systems Concepts Academic Linkage (RASC-AL). Conference May 27, 2011, on the theme of Orbital Debris Mitigation Approaches.

Pusey, Natalie. (2010). The Case of Preserving Nothing: The Need for a Global Response to the Space Debris Problem. Colorado Journal of International Environmental Law and Policy 425 , Spring.

LACHS, Manfred. (1977). El derecho del espacio ultraterrestre. Buenos Aires: Fondo de Cultura Económica.

Williamson, Mark. (2006). Space: The Fragile Frontier. American Institute of Aeronautics and Astronautics, p. 32. Recuperado de la base de datos HeinOnline, el 12 de abril de 2012.

\section{Normatividad}

Interagency Report on Orbital Debris, Office of Science and Technology Policy. November 1995. 
Orbital Debris Quarterly News. Volum. 12. Issue 1, January 2008.

Tratados y principios de las Naciones Unidas sobre el espacio ultraterrestre, 2011.

Investigaciones nacionales sobre la cuestión de los desechos espaciales. Seguridad de los objetos espaciales con fuentes de energía nuclear a bordo y problemas relativos a su colisión con desechos espaciales. Comisión sobre la Utilización del Espacio Ultraterrestre con Fines Pacíficos, Naciones Unidas. A/ AC.105/931.

United Nations Office for Outer Space Affairs. Spece Debris Mitigation Guidelines of the Committee on the Peaceful Uses of Outer Space. 2002.

Inter-Agency Space Debris Coordination Committee IADC Space Debris Mitigation Guidelines. IADC-02-01, Revisión 1, September 2007.

Reporte del Comité sobre la Exploración y Utilización del Espacio Ultraterrestre con Fines Pacíficos 1978-2010.

Inter-Agency Space Debris Coordination Committee. IADC-WD-00-03, Version 4.0. Revision 8, March 12, 2010. p. 13.

United Nations Office for Outer Space Affairs. Main Committee Report of June 15, 2007 as contained in UN Doc. A/62/20 (Annex) pp. 4750. The Space Debris Mitigation Guidelines as adopted by the uncopuos Main Committee were endorsed by the UN General Assembly without a vote in Resolution 62/217 "International Cooperation in the Peaceful Uses of Outer Space" of December 21, 2007.

United Nations. DOC. A/AC-105/C-2/SR, par. 2 (5 de abril de 1979) UN DOC.A/AC-105/C2/L-121)

\section{Páginas de Internet}

http://orbitaldebris.jsc.nasa.gov/library/ IAR_95_Document.pdf Extraído el 12 de mayo de 2012.

http://www.technologyreview.com/computing/18281/ Extraído el 12 de marzo de 2012.

http://orbitaldebris.jsc.nasa.gov/newsletter/ pdfs/ODQNv12i1.pdf Extraído el 19 de noviembre de 2011.

http://www.orbitaldebris.jsc.nasa.gov/library/ IAR_95_Document.pdf Extraído el 10 de noviembre de 2011.

http://webpages.charter.net/dkessler/files/ Collision\%20Frequency.pdf Extraído el 15 de abril de 2012.

http://www.oosa.unvienna.org/pdf/reports/ ac105/AC105_720E.pdf Extraído el 15 de mayo de 2012.

http://www.esa.int/esaMI/Space_Debris/SEM2D7WX3RF_0.html Extraído el 2 de abril de 2012. 
http://www.esa.int/gsp/ACT/ariadna/Ariadna\%20Projects/ARI_study_10-Cfl.html Extraído el 4 de abril de 2012.

http://www.parliament.uk/documents/documents/upload/postpn355.pdf Extraído el 6 de abril de 2012.

http://www.esa.int/esaMI/Space_Debris/SEMQQ8VPXPF_0.html3 Extraído el 15 de marzo de 2012.

http://www.iadc-online.org/index.cgi Extraído el 13 de octubre de 2011.

http://untreaty.un.org/cod/avl/pdf/ha/tos/ tos_s.pdf Extraído el 14 de febrero de 2012.

http://dsostenible.com.ar/situacion-inter/instru-desechosespacial.html Extraído el 22 de febrero de 2012.

ht t p://buscon.rae.es/drael/ SrvltConsulta?TIPO_BUS=3\&LEMA=planeta Extraído el 28 de marzo de 2012.

http://www.arkhaios.com/?p=2989 Extraído el 24 de abril de 2012.

http://www.esa.int/gsp/ACT/ariadna/Ariadna\%20Projects/ARI_study_10-Cfl.html Extraído el 3 de mayo de 2012. http://www.technologyreview.com/computing/18281/ Extraído el 2 de abril de 2012.

http://orbitaldebris.jsc.nasa.gov/library/ IAR_95_Document.pdf Extraído el 13 de marzo de 2012.

h t t p : / / w w w y y u t u b e.co m / watch?v=8iRsaizBUaY Extraído el 17 de mayo de 2012

http://www.au.af.mil/au/awc/awcgate/usspcfs/space.htm Extraído el 16 de abril de 2012.

http://www.nuclearspace.com/ Extraído el 21 de marzo de 2012.

http:/!www.spectrum.ieee.org/WEBONLY/resource/nov02/speak2.htm. Extraído el 24 de mayo de 2012.

http://nuclearfreeplanet.org/blogs/russiasradioactive-phobos-grunt-space-probe-fell-toearth-sunday.html Extraído el 29 de marzo de 2012.

http://space.about.com/od/privatespaceagencies/Private_and_Commercial_Space_Exploration_Agencies.htm Extraído el 21 de mayo de 2012. 